\title{
Thermodynamic scaling of relaxation: insights from anharmonic elasticity
}

\author{
S. Bernini ${ }^{1} \ddagger$, F. Puosi ${ }^{1} \S$, D. Leporini ${ }^{2}$ \\ ${ }^{1}$ Dipartimento di Fisica "Enrico Fermi”, Università di Pisa, Largo B.Pontecorvo 3, I-56127 \\ Pisa, Italy \\ 2 Dipartimento di Fisica "Enrico Fermi”, Università di Pisa, Largo B.Pontecorvo 3, I-56127 \\ Pisa, Italy and IPCF-CNR, UOS Pisa, Italy \\ E-mail: dino.leporinieunipi.it
}

\begin{abstract}
We investigate by Molecular Dynamics simulations of a molecular liquid the thermodynamic scaling (TS) of the structural relaxation time $\tau_{\alpha}$ in terms of the quantity $T \rho^{-\gamma_{t s}}$ where $T$ and $\rho$ are the temperature and the density, respectively. The liquid does not exhibit strong virial-energy correlations. We propose how to evaluate both the characteristic exponent $\gamma_{t s}$ and the TS master curve by resorting to experimentally accessible quantities characterising the anharmonic elasticity and no details about the microscopic interactions. In particular, we express the TS characteristic exponent $\gamma_{t s}$ in terms of the lattice Grüneisen parameter $\gamma_{L}$ and the isochoric anharmonicity $\delta_{L}$. An analytic expression of the TS master curve of $\tau_{\alpha}$ with $\delta_{L}$ as the key adjustable parameter is found. The comparison with the experimental TS master curves and the isochoric fragilities of thirty-four glassformers is satisfying. In a few cases, where thermodynamic data are available, we tested against the available thermodynamic data: i) the predicted characteristic exponent $\gamma_{t s}$, ii) the isochoric anharmonicity $\delta_{L}$, as drawn by the best-fit of TS of the structural relaxation. A linear relation between the isochoric fragility and the isochoric anharmonicity $\delta_{L}$ is found and favourably compared to the experiments with no adjustable parameters. A relation between the increase of the isochoric vibrational heat capacity due to anharmonicity and the isochoric fragility is derived.
\end{abstract}

PACS numbers: 64.70.Q-, 62.10.+s, 07.05.Tp

Keywords: supercooled liquids, elasticity, relaxation, molecular-dynamics simulations

Submitted to: J. Phys.: Condens. Matter

$\ddagger$ Present address: Jawaharlal Nehru Center for Advanced Scientific Research, Theoretical Sciences Unit, Jakkur Campus, Bengaluru 560064, India.

$\S$ Present address: Sciences et Ingénierie des Matériaux et Procédés (SIMaP), UMR CNRS 5266, Grenoble INP, UGA 1130 Rue de la Piscine, BP 75, 38402 Saint-Martin d'Hères Cedex, France 


\section{Introduction}

Temperature-density scaling [1, 2, 3, 4, 5, 6, 7], also known as "thermodynamic scaling" (TS), is an impressive advance in the understanding of the progressive solidification of a supercooled liquid on approaching the glass transition (GT) [8, 9]. TS states that the dependence of the relaxation and the transport of liquids and polymeric systems on the temperature $(T)$ and the density $(\rho)$ may be considered on an equal footing by the scaling quantity $T \rho^{-\gamma_{t s}}$, leading to a material-dependent master curve $[1,2,3,4,5,6,7]$ :

$$
\log \tau_{\alpha}, \log \eta=\mathcal{F}_{T S}\left(T \rho^{-\gamma_{t s}}\right)
$$

where $\tau_{\alpha}$ and $\eta$ are the structural relaxation time and the viscosity, respectively. In Eq.1 both the form of the master curve $\mathcal{F}_{T S}$ and the exponent $\gamma_{t s}$ are system-specific. TS has been usually examined for processes involving very long time scales with a few exceptions [10, 11, 12, 13]. It applies to van der Waals liquids, polymers, ionic liquids [5, 6, 7, 14, 15, 16], liquid crystals [17] and plastic crystals [18] but not to all of the hydrogen-bonded liquids since the equilibrium structure of the liquid and its degree of hydrogen bonding are expected to change when temperature and pressure are changed [19].

TS is attractive for encompassing the changes of both temperature and density so that it represents a severe test of theory and models of the structural arrest occurring at the glass transition (GT). As to the microscopic origin, one hypothesis is that it is strictly related to the intermolecular potential. Indeed, for a liquid having a pairwise additive intermolecular potential described by an inverse power law (IPL) $v(r) \propto r^{-n}$, all the reduced thermodynamic and dynamic properties can be expressed in terms of the variable $\rho^{n / 3} / T$ [20]. Therefore, the conformity of real materials to TS may result from their intermolecular potential being approximated by an IPL, at least in some definite range of intermolecular distance [21]. On a more general ground, Dyre and coworkers proved that liquids with strong correlation of the fluctuations of the virial pressure (W) and the potential energy (U), the so called strongly correlating liquids, exhibit TS. In this scheme the quantity $3 \gamma_{t s}$ is interpreted as the exponent of an effective IPL potential [22, 23, 24]. Even if sufficient, strong virial-energy correlations

are not necessary for TS. Indeed, TS is observed in experiments concerning a few hydrogenbonded liquids (e.g. glycerol and sorbitol) [5] and molecular-dynamics (MD) simulations of supercooled metallic liquids [25] and polymers [13]. All these systems are not strongly correlating liquids since glassformers with competing interactions have poor virial-energy correlations [26, 27]. In particular, polymers exhibit competition between the distinct bonding and non-bonding interactions [13, 28, 29].

Derivations of the TS master curve of the structural relaxation have been reported $[13,30,31,32,33]$. An approach considers the structural relaxation as thermally activated with a density dependent activation energy $\tau_{\alpha}(T, \rho) \sim \exp \left[E_{A}(\rho) / T\right]$ [30]. Assuming $E_{A}(\rho) \propto \rho^{\gamma_{t s}}$, TS is recovered. However, such a picture is in contrast with the fact $\tau_{\alpha}$ is not an exponential function of $T \rho^{-\gamma_{t s}}$ [5]. Casalini et al. used the entropy model of Avramov [34] to derive an expression of the relaxation time in terms of the pressure and the temperature [31, 32]. It accurately fits the experimental data of several glass-forming liquids and polymers with three adjustable parameters, having taken $\gamma_{t s}$ from the experiment. 
Another expression, with the same number of adjustable parameters, based on an entropic model recently formulated by Mauro et al. [35] has been investigated [33]. Very recently, consideration of the TS scaling of the fast mobility, namely the mean square amplitude of the rattling motion of a particle trapped in the cage of the first neighbours, yields a TS master curve of the structural relaxation with two adjustable parameters [13].

Viscous liquids close to GT manifest transient elasticity at short times followed by flow at longer times. As a way to reveal the transitory elastic response in a liquid, a sudden, homogeneous, strain step $\gamma_{x y}$ is applied and the relaxation of the shear stress $\sigma_{x y}(t)$ is followed in time [36]. It is observed that the time-dependent elastic modulus $G(t)=\sigma_{x y}(t) / \gamma_{x y}$ reaches a plateau $G_{p}$ persisting up to the structural relaxation time $\tau_{\alpha}$ [37]. The intermediate-time elastic modulus $G_{p}$ of polymers has the remarkable property, first suggested by Tobolsky in 1960 [38], that it is largely contributed by non-bonding interactions with minor role by stiffer bonds, a feature confirmed by MD simulations [39]. Microscopically, transient elasticity and flow correspond to the temporary trapping of a particle in the cage of the closest neighbours and the subsequent escape process, respectively. The presence of rattling and escape processes in liquids and relationships between them were first proposed by Maxwell [40] and Frenkel [41, 42, 43], see a recent review [44], and later by Tobolsky et al. deriving relations between viscosity and shear modulus [45].

In a solid-like approach of the vibrational properties of a liquid a central role is played by the anharmonicity. Due to anharmonicity, the phonon frequencies depend on the volume and the temperature, as well as the vibration amplitude [44, 46, 47, 48, 49, 50]. Anharmonicity plays an important role in glass formation. Indeed, there is a known correlation between the anharmonicity and fragility $[51,52,53,54,55]$ and it is also shown that the anharmonicity contributes to the fast relaxation [53]. The role of vibrational anharmonicity as key ingredient of the relaxation has been noted $[54,56,57,58]$. In the framework of TS, anharmonicity was also taken into consideration. Some works tried to derive an expression for the TS master curve and explain the meaning of the material dependent exponent basing on the entropy model, the difference between isobaric and isochoric heat capacity and the thermodynamic definition of the Grüneisen parameter $\gamma_{T}$ [31], although the identification of the scaling exponent $\gamma_{t s}$ with the Grüneisen constant is not trivial [59]. For simple liquids it was concluded that $\gamma_{t s} \lesssim 2 \gamma_{T}[60]$.

The presence of elastic modes in supercooled liquids has been considered since long time ago. According to Frenkel [41, 42, 43], the only difference between a liquid and a solid glass is that the liquid does not support all transverse modes as the solid, but only those with frequency $\omega>1 / \tau_{\alpha}$. In the spirit of the Debye theory the number $N_{t}$ of transverse modes with $\omega>1 / \tau_{\alpha}$ in a system with $N$ particles is $N_{t}=2 N\left[1-\left(\omega_{D} \tau_{\alpha}\right)^{-3}\right]$, where $\omega_{D} \sim 10^{13}$ $\mathrm{rad} / \mathrm{s}$ is the Debye frequency [44]. Since in a viscous liquid the relaxation time $\tau_{\alpha}$ fairly exceeds the picosecond timescale, the inequality $\omega_{D} \tau_{\alpha} \gg 1$ follows and $N_{t} \simeq 2 N$, i.e. the number of transverse modes is essentially the same that exist in a solid glass. Consistent with Frenkel's viewpoint, extended, fast modes have been indicated in several studies concerning liquids $[61,62,63,64,65,66,67,68]$. Even in the presence of local order [69, 70], the major role of the extended elastic modes in setting the mean square amplitude of the rattling motion 
of a trapped particle in the cage of the neighbours has been revealed [71, 72].

The prominent role of elasticity in the relaxation process is investigated by the so-called elastic models, see refs. [73, 74] for excellent reviews and refs. [75, 76, 77, 55, 78, 79, $80,44,81,82,83,84,85,86,37,87,39,88]$ for recent related papers. In particular, both MD simulations [37, 87] and extended comparison with experiments [87] suggest strong correlation between the structural relaxation and transient elasticity. The related master curve has the form [37, 87]:

$$
\log \tau_{\alpha}=\widetilde{\Upsilon}_{0}+\widetilde{\Upsilon}_{1} X+\widetilde{\Upsilon}_{2} X^{2}
$$

where

$$
X=\frac{G_{p} T_{g}}{G_{p g} T}
$$

$G_{p g}$ is the modulus at the glass transition, $\widetilde{\Upsilon}_{1}=3.4(2), \widetilde{\Upsilon}_{2}=10.3(8)$ are suitable universal constants independent of the kinetic fragility, and $\widetilde{\Upsilon}_{0}=2-\widetilde{\Upsilon}_{1}-\widetilde{\Upsilon}_{2}$ to comply with the usual definition $\tau_{\alpha}=100 \mathrm{~s}$ at the glass transition. Eq. 2 has been validated in the whole supercooled region by MD simulations on polymers [37] and ionic liquids [87], as well as comparison with the experimental data concerning several glassformers in a wide fragility range $(20 \leq m \leq 115)$ including van der Waals and hydrogen-bonded liquids, metallic glasses and the strong glassformer silica (the latter over about fifteen decades of viscosity with no adjustable parameters) [87]. Eq.2 follows from an elastic cavity model [87], relating the linear elasticity with the fast mobility, and consideration of the universal master curve between the latter and the structural relaxation [89, 90, 91, 92, 93, 94, 95, 12, 96, 97, 71, 67].

Building on the elastic scaling of the structural relaxation, Eq. 2, we interpret TS as related to the temperature/density dependence of the shear elasticity due to anharmonicity. The study examines the matter by Molecular-Dynamics (MD) simulations of a model molecular liquid and compares the MD findings with the available experimental data. The model under study exhibits limited changes of the local structures in virtue of the high packing of the investigated states [69, 71, 98]. Notably, our model liquid does not manifest strong virial-energy correlations [24], as previously noted [28, 13] and recently reported for a very similar model [29].

The paper is organized as follows: Sec.2 illustrates basic results about anharmonic elasticity, Sec.3 gives details about the MD simulations, Sec.4 discusses the results of the MD simulations and the comparison with the experimental data. Finally, Sec.5 summarizes the conclusions. 


\section{Anharmonic elasticity}

\subsection{General aspects}

The vibrational frequencies in harmonic solids do not depend on either the density or the temperature since the normal frequencies have no dependence on volume or strain [46]. In addition, purely harmonic solids do not exhibit thermal expansion and temperature dependence of the elasticity [47]. Real solids are never purely harmonic since the general conditions to ensure the absence of terms beyond the second order in the displacements from the lattice potential energy are rather stringent [48].

To deal with anharmonic response, we consider vibrational modes with frequency $\nu_{i}$. Under infinitesimal changes of temperature and density one has:

$$
d \ln \nu_{i}=\gamma_{i} d \ln \rho-\delta_{i} d \ln T
$$

where $\gamma_{i}$ is the isothermal Grüneisen parameter [99, 49]:

$$
\gamma_{i}=-\left.\frac{\partial \ln \nu_{i}}{\partial \ln V}\right|_{T}
$$

and $\delta_{i}$ is defined as:

$$
\delta_{i}=-\left.\frac{\partial \ln \nu_{i}}{\partial \ln T}\right|_{V}
$$

The parameter $\delta_{i}$ accounts for the isochoric changes of frequency and is recast as:

$$
\delta_{i}=-T a_{i}
$$

where the (intrinsic) isochoric mode anharmonicity $a_{i}$ is defined as [100]:

$$
a_{i}=\left.\frac{\partial \ln \nu_{i}}{\partial T}\right|_{V}
$$

It can be shown that $[100,101]$ :

$$
a_{i}=\alpha\left(\gamma_{i}-\gamma_{i P}\right)
$$

where $\alpha$ is the thermal expansion coefficient

$$
\alpha=-\left.\frac{\partial \ln \rho}{\partial T}\right|_{P}
$$

and the isobaric Grüneisen parameter of the i-th mode is defined as:

$$
\gamma_{i P}=-\left.\frac{\partial \ln \nu_{i}}{\partial \ln V}\right|_{P}
$$

Because the measured isobaric parameters are in general higher than the isothermal ones, the $a_{i}$ parameters are usually negative so that $\delta_{i}>0$, namely in an isochoric experiment the mode frequency often decreases with increasing temperature.

Eq.4 incorporates two distinct kind of anharmonicity, the extrinsic anharmonicity, i.e., volume-dependent properties such as the Grüneisen parameter and the related thermal expansion, as well as the intrinsic anharmonicity yielding temperature-dependence at constant volume (corresponding to explicit temperature dependence of the vibrational frequencies) [102]. Extrinsic anharmonicity is usually managed by quasi-harmonic approximation (QHA) 


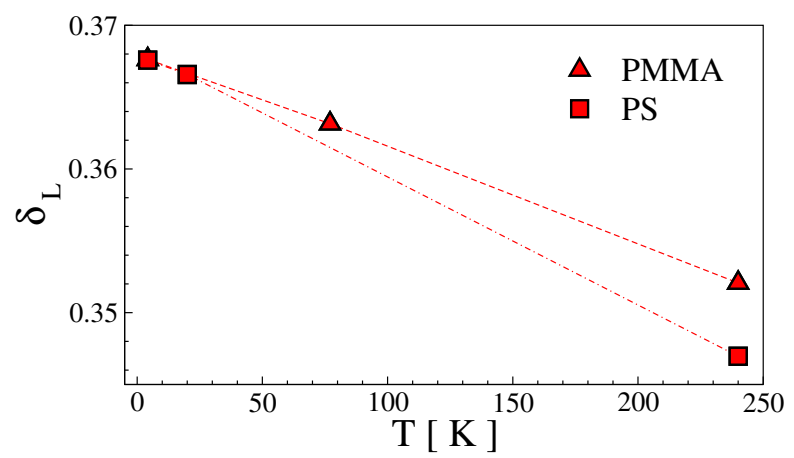

Figure 1. Isochoric-anharmonicity parameter of glassy PMMA and PS [103].

[102]. In the QHA approximation, it is assumed that the solid behaves like a harmonic solid at given volume, i.e. phonons are well-defined, but the phonon frequencies depend on volume. The frequencies do not depend explicitly on temperature, so that heating at constant volume does not change them. QHA yields $\delta_{i}=0$, i.e. $\gamma_{i}=\gamma_{i P}$ according to Eq.7 and Eq. 9 [100]. Like in the harmonic approximation, the energy needed to remove an atom from the crystal is infinite in QHA - therefore, diffusion and melting cannot be explained within this approximation [50]. QHA breaks down at high temperatures where phonon-phonon interactions and explicit temperature dependence of the vibrational frequencies become prominent.

\subsection{Lattice Grüneisen parameter $\gamma_{L}$}

Our major interest will be limited to long-wavelength acoustic modes. We will not make distinction between transverse and longitudinal modes, i.e. all the acoustic frequencies are taken with the same volume dependence and then equal Grüneisen parameter. In the Debye model [99] this assumption results in a volume-independent Poisson ratio [46]. The Grüneisen parameter restricted to the acoustic modes is usually referred to as lattice Grüneisen parameter [104]. To make it explicit, henceforth, we set the subscript $i=L$ from Eqs. 4 through Eq. 11. The lattice Grüneisen parameter $\gamma_{L}$ is dominated by the intermolecular forces $[105,106,107]$. In contrast, the usual thermodynamic Grüneisen parameter $\gamma_{T}$ is contributed by both intra- and inter-molecular interactions [99]. For metals and ionic crystals $\gamma_{T} \simeq \gamma_{L}$ [104]. However, for molecular systems a large difference between these two Grüneisen parameters can exist. This is due to the very different values of the intra- and inter-molecular Grüneisen parameters and their relative weight at different temperatures. Normally, intrachain covalent bonds vibrations (high frequency, short wave length) have very low values of the Grüneisen parameter, while interchain anharmonic vibrations involving van der Waals bonds (low frequency, long wave length) have very high values of the Grüneisen parameter. $\gamma_{L}$ is affected by the local order [106] and is a weak function of temperature [107, 104, 108] with no (see poly(chlorotrifluoroethylene) in Ref. [104]) or small [109] step across the glass transition, with the notable exception of poly(vinyl acetate) [108]. In contrast, $\gamma_{T}$ is strongly temperature dependent. At low temperature, where only acoustic inter-molecular modes are 
excited, $\gamma_{T} \sim \gamma_{L}$, whereas $\gamma_{T} \ll \gamma_{L}$ at high temperature where optical, quasi-harmonic vibrations dominate [110]. The ratio $\gamma_{L} / \gamma_{T}$ is related to the ratio of the total heat capacity at constant volume $C_{V}$ and the intermolecular contribution $C_{\text {inter }}[105,106,104]$ :

$$
\frac{\gamma_{L}}{\gamma_{T}}=\frac{C_{V}}{C_{\text {inter }}}
$$

At high temperature $\gamma_{T} \sim 1$ and $C_{\text {inter }} / C_{V}$ is of the order of $10-20 \%$, so that $\gamma_{L} / \gamma_{T} \sim 5$ to 10 , e.g. $\gamma_{L}=4.0$ for poly(methylmethacrylate) and $\gamma_{L}=9.0$ for isotactic poly(propylene) [104].

\subsection{Isochoric anharmonicity $\delta_{L}$}

The isochoric anharmonicity parameter $\delta_{L}$, defined by Eq.6 with $i=L$, if treated perturbatively, is mainly affected by cubic and quartic anharmonicity associated to threephonon and four-phonon processes, respectively [111]. $\delta_{L}$ changes mildly across the glassy regime of several polymeric glassformers [103], see Fig.1 for two selected examples. Fig.1 suggests that under isochoric conditions $\nu \sim T^{-\delta_{L}}$ with nearly constant $\delta_{L}$. We may figure out that the vibrational mode with frequency $\nu$ follows by a suitable effective confining potential $u_{\text {eff }}(q)$ where $q$ is a generalized coordinate. Assuming $u_{\text {eff }}(q) \propto q^{\beta}$ with $\beta>0$ yields $\nu \propto E^{1 / 2-1 / \beta}$ where $E$ is the associated total energy [112]. With $E \sim\langle E\rangle \sim T$ one obtains:

$$
\delta_{L}=\frac{1}{\beta}-\frac{1}{2}
$$

For harmonic potential $(\beta=2), \delta_{L}=0$ as expected, whereas $\delta_{L}>0$ implies $\beta<2$. In particular, with $\delta_{L} \sim 0.36$, see Fig.1, one has $\beta \sim 1.16$ suggesting moderate anharmonicity in the glassy phase of polymers.

For molecular liquids the parameter $\delta_{L}$ has been derived as [101]:

$$
\delta_{L}=1-\alpha T-\frac{1}{n}
$$

Eq.14 predicts $\delta_{L}<1$ and relies on the modified Van der Waals equation of state due to Flory which deals with both polymers [113] and liquids [114]. The positive parameter $n$ is the ratio between the inner pressure $P_{i}$ and the cohesive energy density c.e.d. $[115,116]$

In a solid-state approach the isochoric anharmonicity parameter $\delta_{L}$ may be related to the constant volume heat capacity. To show that, we start from the expression [117]:

$$
C_{V}^{v i b}=3 N k_{B}\left[1-\frac{2 T}{\bar{\nu}} \frac{d \bar{\nu}}{d T}+\frac{T^{2}}{\bar{\nu}^{2}}\left(\frac{d \bar{\nu}}{d T}\right)^{2}-\frac{T^{2}}{\bar{\nu}} \frac{d^{2} \bar{\nu}}{d T^{2}}\right]
$$

where $k_{B}$ and $\bar{\nu}$ are the Boltzmann constant and the geometrically averaged phonon frequency, respectively and the derivatives are taken at constant volume. Eq.15 is derived by the free energy of a harmonic solid in the high-temperature approximation and takes into account the isochoric frequency change with the temperature due to the intrinsic anharmonicity. When extended to viscous liquids, Eq.15 represents the total heat capacity, i.e. $C_{V} \simeq C_{V}^{v i b}$, if the configurational contribution $C_{V}^{\text {conf }}$ is negligible [117, 44]. Reminding the relation $C_{V}=T \partial S /\left.\partial T\right|_{V}$, support to the inequality $C_{V}^{\text {conf }} \ll C_{V}^{v i b}$ is offered by liquid silica where 
the configurational entropy is much smaller than the vibrational entropy [118]. By plugging Eq. 6 with $i=L$ in Eq.15 and neglecting the temperature dependence of $\delta_{L}$ we get:

$$
C_{V}^{v i b}=3 N k_{B}\left(1+\delta_{L}\right)
$$

The breakdown of the Dulong-Petit law which is seen in Eq.16 is a known effect of the anharmonicity [44]. An alternative treatment of the isochoric anharmonicity derives the relation $d \ln \bar{\nu} /\left.d T\right|_{V}=-\alpha / 2(q-1)$, where $q$ is the logarithmic derivative of the average Grüneisen parameter with respect to volume, and recasts Eq. 15 as [119]:

$$
C_{V}^{v i b}=3 N k_{B}[1+\alpha(q-1) T]
$$

In the present context $T \simeq T_{g}$. Referring to Eq. 7 and Eq. 9, we see that Eq. 16 and Eq. 17 yield similar results provided that $\gamma_{L P} \simeq \gamma_{L}+q-1$. Usually $q \sim 2$ [119]. Further comparison between Eq.16 and Eq.17 is deferred to Sec.4.4.3.

\subsection{Anharmonic modulus}

We now restrict Eq. 4 to acoustic shear waves and assume $\gamma_{L}$ and $\delta_{L}$ as constant quantities. Integration of Eq. 4 and consideration of the dispersion law $\omega=\left(G_{p} / \rho\right)^{1 / 2} k$ yields the temperature-density scaling of the shear modulus $G_{p}$ :

$$
G_{p}=A \frac{\rho^{2 \gamma_{L}+1 / 3}}{T^{2 \delta_{L}}}
$$

where $A$ is a constant. Under isothermal conditions Eq.18 is an alternative form of the socalled Birch's law relating the velocity of elastic waves to density [120]. Under isobaric conditions Eq.10 and Eq.18 yield

$$
G_{p}(T) \sim \exp \left[-C\left(\frac{T}{T_{g}}\right)\right]
$$

where, in the QHA spirit, the weak power-law dependence due to the isochoric anharmonicity has been neglected. The parameter $C$ is given by

$$
C=\left(2 \gamma_{L}+1 / 3\right) \alpha T_{g}
$$

All the quantities in the right hand side of Eq.20 are attainable by experiments. For polymers $\alpha T_{g} \simeq 0.164$ [121] and $\gamma_{L}$ is in the range $4-10[104,107]$ so that $1.4 \lesssim C \lesssim 3.3$. The exponential decay of the elastic modulus with temperature, Eq.19, has been considered in supercooled metallic melts $[122,86]$. In particular, Krausser et al find $C=(2+\lambda) \alpha T_{g}$ [86] where $\lambda$, the steepness of the short-ranged repulsion, is a fitting parameter, whereas Harmon et al considered $C$ as fitting parameter [122]. In supercooled metallic melts $C$ is found in the range $0.6-3.3$ [86] as well as $C=1.29$ [122]. The fact that the parameter $C$ controlling the temperature influence on the elastic modulus under isobaric conditions is quite similar in polymers and supercooled metallic melts suggests similarities between their extrinsic anharmonicities. Finally, we note that the lattice Grüneisen parameter $\gamma_{L}$ is also affected by the steepness of the short-ranged repulsion [106], so that Eq.20 is consistent with the findings of Krausser et al [86] . 


\section{Methods}

A coarse-grained polymer model of a melt of 667 linear, unentangled chains with three monomers per chain is considered. The chains are fully-flexible, i.e. bond-bending and bondtorsions potentials are not present. Non-bonded monomers at distance $r$ belonging to the same or different chains interact via the truncated Lennard-Jones (LJ) potential:

$$
U_{L J}(r)= \begin{cases}\varepsilon\left[\left(\frac{\sigma^{*}}{r}\right)^{12}-2\left(\frac{\sigma^{*}}{r}\right)^{6}\right]+U_{c u t} & \text { if } r \leq r_{c u t} \\ 0 & \text { otherwise }\end{cases}
$$

where $\sigma^{*}=\sqrt[6]{2} \sigma$ is the position of the potential minimum with depth $\varepsilon$, and the value of the constant $U_{c u t}$ is chosen to ensure $U_{L J}(r)=0$ at $r=r_{c u t}=2.5 \sigma$. The bonded monomers interact by a stiff potential $U_{b}$ which is the sum of the LJ potential and the FENE (finitely extended nonlinear elastic) potential [123]:

$$
U_{F E N E}(r)=-\frac{1}{2} k R_{0}^{2} \ln \left(1-\frac{r^{2}}{R_{0}^{2}}\right)
$$

$k$ measures the magnitude of the interaction and $R_{0}$ is the maximum elongation distance. The parameters $k$ and $R_{0}$ have been set to $30 \varepsilon / \sigma^{2}$ and $1.5 \sigma$ respectively [124]. The resulting bond length is $r_{b}=0.97 \sigma$ within a few percent. All quantities are in reduced units [125]: length in units of $\sigma$, temperature in units of $\varepsilon / k_{B}$ and time $\tau_{M D}$ in units of $\sigma \sqrt{m / \varepsilon}$ where $m$ is the monomer mass. We set $m=k_{B}=1$ Periodic boundary conditions are used. $N V T$ ensemble (constant number of particles, volume and temperature) has been used for equilibration runs, while $N V E$ ensemble (constant number of particles, volume and energy) has been used for production runs for a given state point. The simulations were carried out using LAMMPS molecular dynamics software (http://lammps.sandia.gov) [126]. Several states with different number density $\rho$ and temperature $T$ are studied. Different time steps are chosen in order to ensure an energy drift of about $1 \%$ in NVE runs. In particular, the following $\left[\rho ; T_{1}, T_{2}, \ldots\right]$ pairs are simulated with time step $3 \cdot 10^{-3}$ : [0.98; 0.5], [0.984; 0.3, 0.31, 0.32, 0.33, 0.34, $0.36,0.4,0.5,0.6],[0.995 ; 0.6],[1 ; 0.4,0.7],[1.015 ; 0.6],[1.020 ; 0.5,0.7],[1.033 ; 0.4,0.45$, $0.5,0.55,0.6,0.65,0.7,0.8,0.9],[1.035 ; 0.6],[1.039 ; 0.5],[1.04 ; 0.7],[1.05 ; 0.5],[1.052$; $0.5],[1.055 ; 0.6],[1.056 ; 0.7],[1.071 ; 0.7],[1.075 ; 0.6],[1.086 ; 0.6,0.63,0.7,0.75,0.8$, $0.9,1],[1.09 ; 0.7,0.75,0.8],[1.095 ; 0.6],[1.1 ; 0.95]$. The following $\left[\rho ; T_{1}, T_{2}, \ldots\right]$ pairs are simulated with time step $1 \cdot 10^{-3}:[1 ; 1,1.2,1.4,1.6]$, [1.05; 1.3, 2], [1.025; 1.44, 2.02, 2.6].

For each state we averaged over at least sixteen different runs. The equilibration

procedure involves runs with time lengths $\Delta t_{e q}$ exceeding at least three times the average reorientation time of the end-end vector [127].

\section{Results and discussion}

\subsection{General Aspects}

4.1.1. Mobility, relaxation and elastic scaling We define the monomer displacement in a time $t$ as:

$$
\Delta \mathbf{r}_{i}(t)=\mathbf{r}_{i}(t)-\mathbf{r}_{i}(0)
$$




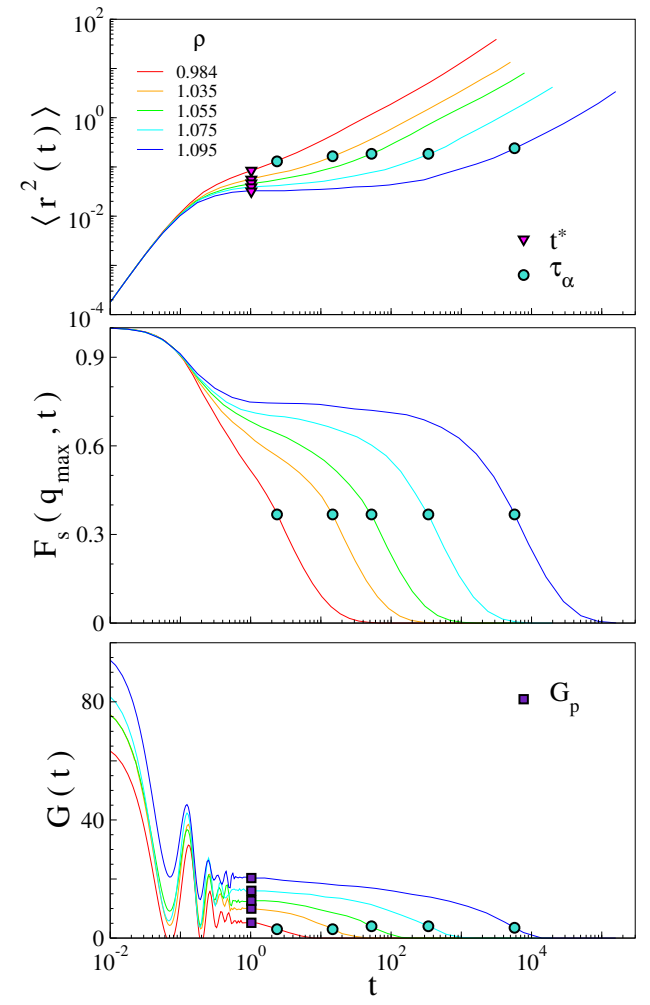

Figure 2. Mobility, relaxation and elastic response according to the MD simulations of the liquid of linear trimers at $T=0.6$ and different densities. Monomer MSD, Eq.24 (top), corresponding ISF, Eq.25 (middle), elastic response (bottom). The triangles, dots and squares mark the positions of the inflection point in the MSD $\left(t^{*}\right)$, the relaxation time $\left(\tau_{\alpha}\right)$ and the height of the plateau of the elastic response $\left(G_{p}\right)$, respectively. All the quantities are in reduced MD units.

where $\mathbf{r}_{i}(t)$ is the vector position of the $i$-th monomer at time $t$. The mean square displacement (MSD) $\left\langle r^{2}(t)\right\rangle$ is expressed as:

$$
\left\langle r^{2}(t)\right\rangle=\left\langle\frac{1}{N} \sum_{i=1}^{N}\left\|\Delta \mathbf{r}_{i}(t)\right\|^{2}\right\rangle
$$

where brackets denote the ensemble average and $N$ is the total number of monomers. In addition to MSD the incoherent, self part of the intermediate scattering function (ISF) is also considered:

$$
F_{s}(q, t)=\left\langle\frac{1}{N} \sum_{j=1}^{N} e^{i \mathbf{q} \cdot \Delta \mathbf{r}_{j}(t)}\right\rangle
$$

ISF was evaluated at $q=q_{\max }$, the maximum of the static structure factor $\left(7.06 \leq q_{\max } \leq\right.$ 7.35 ). Fig.2 shows illustrative examples of the monomer MSD (top) and ISF (middle). At very short times (ballistic regime) MSD increases and ISF starts to decay. At later times, when the temperature is lowered and/or the density is increased, a quasi-plateau region occurs in both MSD and ISF, and an inflection point is seen at $t^{*} \simeq 1.023$ in the log-log MSD plot, see Fig. 2 (top). $t^{*}$ is state-independent in the present model [89]. The inflection point signals 


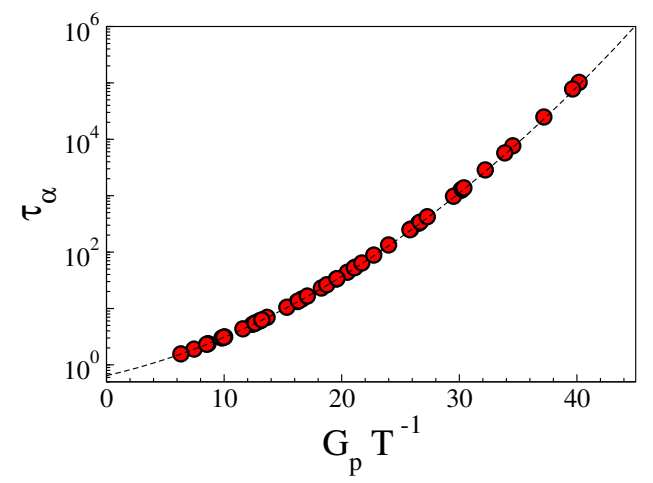

Figure 3. Elastic master curves of the relaxation time $\tau_{\alpha}$ according to the MD simulations. The dashed line is Eq.30 with $\Upsilon_{0}=-0.191(8), \Upsilon_{1}=0.048(3), \Upsilon_{2}=0.0020(1)$ [37, 87]. No adjustable parameters are allowed. All the quantities are in reduced MD units.

the end of the exploration of the cage by the trapped particle and the subsequent early escapes. We define the fast mobility of the monomers of the linear chains as the MSD at $t^{*}$ [89]:

$$
\left\langle u^{2}\right\rangle=\left\langle r^{2}\left(t=t^{*}\right)\right\rangle
$$

The fast mobility is the mean square amplitude of the position fluctuations of the tagged particle in the cage of the neighbours. The inflection point in the log-log MSD plot disappears if $\left\langle u^{2}\right\rangle>\left\langle u_{m}^{2}\right\rangle=0.125$ signalling the absence of significant cage effect by the neighbours of the tagged particle. The structural relaxation time $\tau_{\alpha}$, the average escape time from the cage, is defined by the relation $F_{s}\left(q_{\max }, \tau_{\alpha}\right)=e^{-1}$.

The off-diagonal $x y$ component of the stress tensor is defined by [37]:

$$
\sigma_{x y}=\frac{1}{V}\left[\sum_{i=1}^{N}\left(m v_{x, i} v_{y, i}+\frac{1}{2} \sum_{j \neq i} r_{x, i j} F_{y, i j}\right)\right]
$$

where $V=N / \rho$ is the volume of the system, $v_{\alpha, i}$ is the $\alpha$ component of the velocity of the $i$-th monomer, $r_{\alpha, i j}$ is the $\alpha$ component of the vector joining the $i$-th monomer with the $j$-th one and $F_{\alpha, i j}$ is the $\alpha$ component of the force between the $i$-th monomer and the $j$-th one. The shear stress correlation function is defined by [128]:

$$
G_{x y}(t)=\frac{V}{k_{B} T}\left\langle\sigma_{x y}\left(t_{0}\right) \sigma_{x y}\left(t_{0}+t\right)\right\rangle
$$

where the brackets $\langle\ldots\rangle$ denote the canonical average. The average value of $G_{x y}(t), G_{y z}(t)$ and $G_{z x}(t)$ will be denoted as $G(t)$. Fig.2 (bottom) plots the modulus $G(t)$. At short times $(t \lesssim 0.5) G(t)$ is characterized by oscillations due to the bond length fluctuations [39]. For longer times ( $t \gtrsim 0.5)$ the oscillations of $G(t)$ vanish, force equilibration takes place [37], and the modulus $G(t)$ approaches a plateau-like region. The persistence of the elastic response is due to the cage effect, namely the trapping period of each monomer in the cage of the first neighbours which is terminated by the structural relaxation time $\tau_{\alpha}$ [129]. Beyond $\tau_{\alpha} G(t)$ relaxes according to the polymer viscoelasticity. We are not interested here in this long-time 


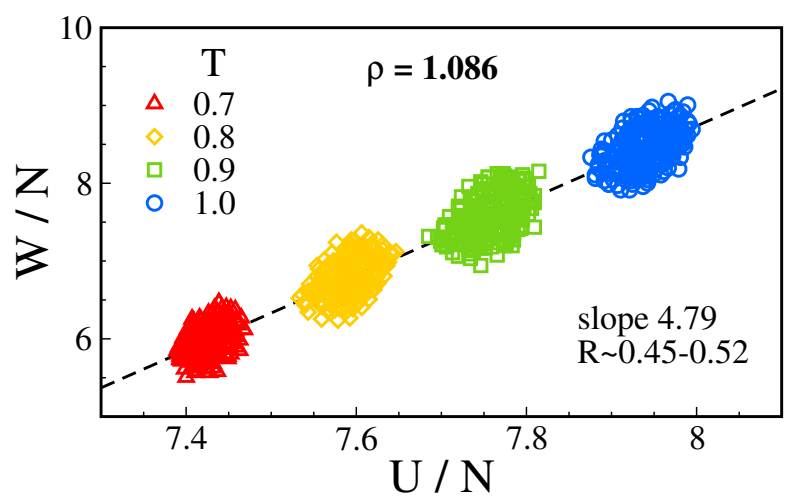

Figure 4. Correlation plot of the virial and the configurational energy per particle from MD simulations of states with same density and different temperatures. All the quantities are in reduced MD units.

decay which has been addressed by other studies [130]. We define the finite frequency shear modulus $G_{p}$ as [37, 87, 39]:

$$
G_{p} \equiv G\left(t^{*}\right)
$$

Fig.3 shows the elastic scaling of the structural relaxation (top) and the cage rattling amplitude (bottom) in agreement with other studies [37, 87]. For MD simulations it is convenient to recast Eq.2 as [37, 87]:

$$
\log \tau_{\alpha}=\Upsilon_{0}+\Upsilon_{1}\left(\frac{G_{p}}{T}\right)+\Upsilon_{2}\left(\frac{G_{p}}{T}\right)^{2}
$$

4.1.2. Virial-energy correlations In the case of pair potentials, the virial $W$, i.e., the configurational contribution to pressure, is given by [131]:

$$
W=-\frac{1}{3} \sum_{i>j} w\left(\left|\mathbf{r}_{i}-\mathbf{r}_{j}\right|\right)
$$

where $w(r)=r v^{\prime}(r), v^{\prime}$ being the derivative of the pair potential $v(r)$. For an IPL potential, $v(r) \propto r^{-n}$, one has $w(r)=-n v(r)$ and the virial is proportional to the potential energy $U=\sum_{i>j} v\left(\left|\mathbf{r}_{i}-\mathbf{r}_{j}\right|\right)$ :

$$
W=\frac{n}{3} U
$$

Eq.32 states that in IPL systems, irrespective of the physical state, the scatter plot of the instantaneous potential energy and virial shows perfect correlation with slope $n / 3$. As pointed out in the Introduction, liquids with strong virial-energy correlations exhibits TS with $\gamma_{t s}=n / 3[22,23,24]$. Figure 4 plots the instantaneous virial and potential energy fluctuations of the liquid of trimers according to our MD simulations. The degree of correlation is quantified by the correlation coefficient $R$ :

$$
R=\frac{\langle\Delta W \Delta U\rangle}{\sqrt{\left\langle(\Delta W)^{2}\right\rangle} \sqrt{\left\langle(\Delta U)^{2}\right\rangle}}
$$



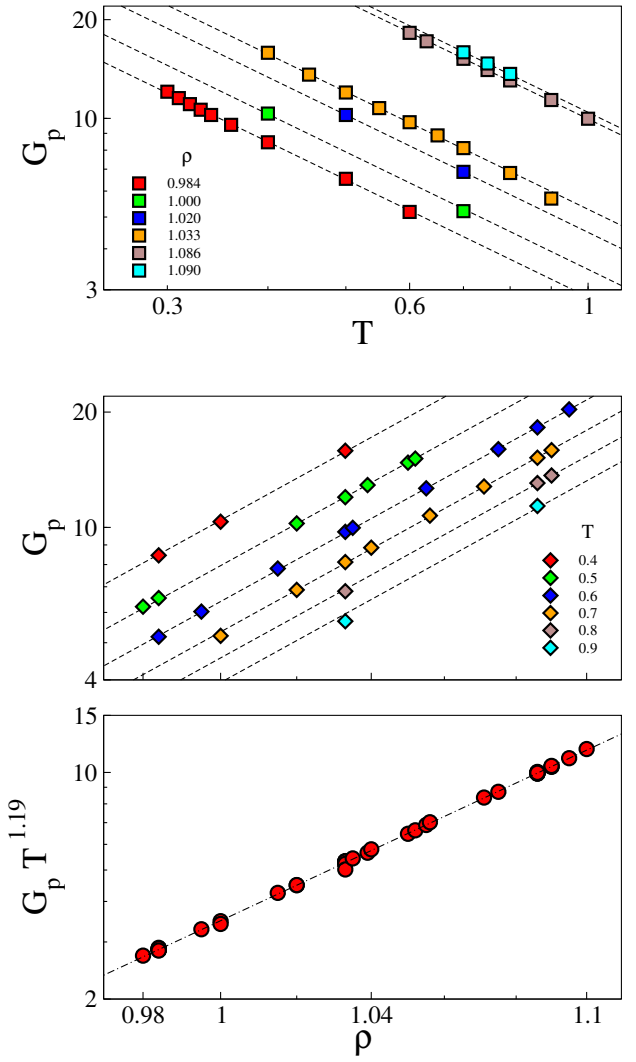

Figure 5. Isochoric temperature dependence (top) and isothermal density dependence (middle) of the shear elastic modulus $G_{p}$ for the melt of linear trimers. All the lines of the top and the middle panels have slope -1.19 and 12.71, respectively. Bottom: temperature-density scaling of the elastic modulus. The dot-dashed line is Eq.18 with $A=3.489, \gamma_{L}=6.19$ and $\delta_{L}=0.595$. All the quantities are in reduced MD units.

where $\Delta$ denotes the deviation from the average value of the given quantity and $\langle\ldots\rangle$ denotes the thermal averages. We find low correlation, $R \sim 0.45-0.52$, depending on the state. In contrast, in the case of RB chains the correlation is high, $R>0.8$ (not shown), as in previous studies on linear chains with rigid bonds [28, 132]. The drop of the virialenergy correlations by replacing rigid bonds with semirigid ones in linear chains has been noted $[28,29,13]$.

\subsection{Temperature-density scaling of the elastic response}

Fig.5 shows that, in the range of interest, the elastic modulus exhibits power-law dependence on both the temperature (top panel) and the density (middle panel). The combined master curve is of the form predicted by Eq.18 (bottom panel) with $\gamma_{L}=6.19$ and $\delta_{L}=0.595$. The best-fit value $\gamma_{L}=6.19$ is within the range of the reported experimental values and close to, e.g., $\gamma=6.1$ in poly(chlorotrifluoroethylene) and $\gamma=6.4$ in poly(vinylidene fluoride) and polyethylene (low density) [104]. 


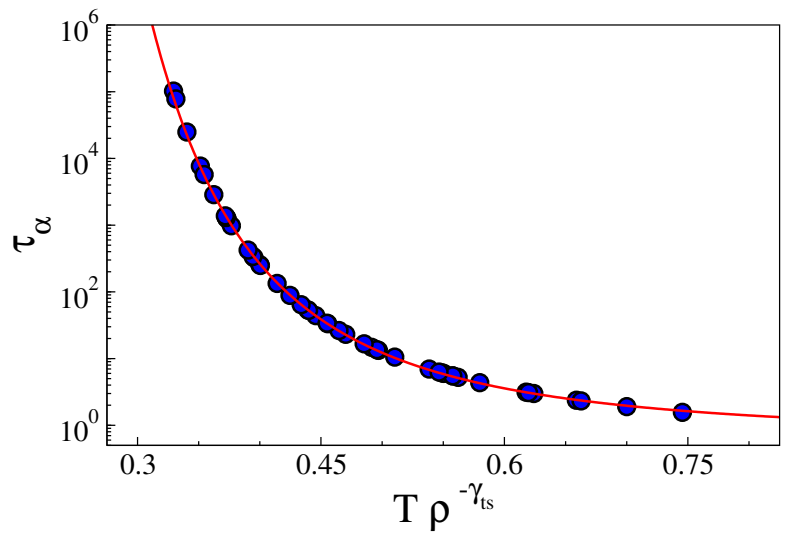

Figure 6. TS master curve of the structural relaxation according to the MD simulations of the linear trimers with $\gamma_{t s}=5.80(1)$, as drawn by Eq.34 with data from Fig.5. The red line is the combination of Eq.30 with parameters in Fig.3 and Eq.18 with parameters in Fig.5. No adjustment is done. All the quantities are in reduced MD units.

\subsection{Temperature-density scaling of the structural relaxation}

The temperature-density dependence of the elastic modulus, Eq.18, and the elastic scaling in $G_{p} / T$ of the structural relaxation, Eq.30, suggest that the TS characteristic exponent is given by:

$$
\gamma_{t s}=\frac{2 \gamma_{L}+1 / 3}{2 \delta_{L}+1}
$$

For the liquid of trimers under study Eq.34 yields $\gamma_{t s}=5.80$ with $\gamma_{L}=6.19$ and $\delta_{L}=0.595$, according to Fig.5. Fig.6 presents the TS scaling of the structural relaxation of all the investigated states by the MD simulations and tests Eq.34. It shows that $\gamma_{t s}=5.80$ collapses all the data concerning the structural relaxation time in a well-defined master curve.

\subsection{Comparison with the experiments}

This Section compares the findings of the MD simulations with a wide set of experimental data concerning TS of the structural relaxation. The task is rather ambitious in that the experimental TS extends up to relaxation times as long as $\sim 10^{2} \mathrm{~s}$, whereas MD simulations are limited to fractions of microseconds. The comparison with the experiments is twofold. First, we test Eq.34 on a few glassformers. The limited set of systems scrutinized is due to the scarcity of experimental data, especially concerning $\delta_{L}$. Second, we extensively compare the prediction concerning the TS master curve of the structural relaxation with thirty-four different glassformers.

4.4.1. Test of Eq.34 Table 1 provides the predicted scaling exponent $\gamma_{t s}^{P W}$ according to Eq.34 for some glassformers, i.e. the polymers poly(methyl methacrylate) (PMMA) and polystyrene (PS), and the molecular liquid o-tephenyl (OTP). Consideration of further systems was hampered by the absence of available data concerning $\gamma_{L}$ and/or $\delta_{L}$. Particular care was 
Table 1. Comparison between the experimental value of the TS exponent $\gamma_{t s}^{e x p}$ and the predictions of both the present work $\left(\gamma_{t s}^{P W}\right)$, Eq.34, and the one of Ref.[7] $\left(\gamma_{t s}^{C R}\right)$ for the polymers poly(methyl methacrylate) (PMMA) and polystyrene (PS) and the molecular liquid o-tephenyl (OTP). The physical quantities used to calculate $\gamma_{t s}^{P W}$ are listed. MD data are also listed for comparison. $\delta_{L g}$ is the anharmonicity parameter, Eq.6 with $i=L$, evaluated at $T_{g}$. For PMMA and PS $\delta_{L}=\alpha_{l} / \alpha_{g} \delta_{L g}$. For OTP $\delta_{L}$ was evaluated at $T_{g}$ via Eq.14. See text for further details.

\begin{tabular}{ccccccccc}
\hline \hline & $T_{g}(\mathrm{~K})$ & $\delta_{L g}{ }^{a}$ & $\frac{\alpha_{l}}{\alpha_{g}}$ & $\delta_{L}$ & $\gamma_{L}$ & $\gamma_{t s}^{P W}$ & $\gamma_{t s}^{C R} b$ & $\gamma_{t s}^{\text {exp } b}$ \\
\hline \hline MD & & & $1.75^{c}$ & 0.595 & 6.19 & 5.8 & & \\
PMMA & 378 & 0.34 & $1.96^{d}$ & 0.67 & $4.0^{e}$ & 3.6 & $2.8 \pm 0.34$ & $1.8^{f}$ \\
PS & 373 & 0.334 & $2.75^{d}$ & 0.92 & $4.4^{e}$ & 3.2 & $2.1 \pm 0.3$ & 2.5 \\
OTP & 243 & & & 0.32 & $3.3^{g}$ & 4.2 & $4.05 \pm 0.3$ & 4 \\
\hline \multicolumn{10}{c}{}
\end{tabular}

${ }^{a}$ Ref.[103]

${ }^{b}$ Ref.[7]

${ }^{c}$ Ref.[133]

${ }^{d}$ Ref.[121]

${ }^{e}$ Ref.[104]

${ }^{f} \gamma_{t s}^{P M M A}=1.94$ according to more accurate data [134]

${ }^{g}$ from Brillouin light scattering at $0.95 T_{g}$ [135]

devoted to derive the parameter $\delta_{L}$. For polymers, we extrapolated at $T_{g}$ the experimental data in the glassy state, see Fig.1, and multiplied the resulting value for the ratio of the expansion coefficients in the liquid and the glassy state $\alpha_{l} / \alpha_{g}$. The procedure relies on the assumption that the major contribution to the changes of $\delta_{L}$ from the glassy to the liquid regime are due to the increased thermal expansion, see Eq.7 and Eq. 9 since, as noted in Sec.2, the lattice Grüneisen parameter of polymers are weakly affected by the glass transition [107, 104]. For OTP, to the best of our knowledge, no data are available to derive $\delta_{L}$ according to the procedure adopted for polymers. As an alternative, we resorted to Eq.14 with $\alpha T_{g}=0.182$ [136] and $n=2$, as expected for Van der Waals liquids and found for benzene [137].

Table 1 compares $\gamma_{t s}^{P W}$, Eq.34, with the experimental value of the TS exponent $\gamma_{t s}^{\text {exp }}$ and the prediction according to Ref.[7] $\left(\gamma_{t s}^{C R}\right)$. The results are encouraging but less accurate than the ones by the thermodynamic method of Ref.[7]. We notice that the MD results and the experimental values for PMMA and PS concerning the anharmonicity, i.e. the ratio $\alpha_{l} / \alpha_{g}, \delta_{L}$ and $\gamma_{L}$, are rather close, suggesting that the MD model, even if rather crude, captures the key aspects. We find that $\gamma_{t s}^{P W}$, Eq.34, overestimates $\gamma_{t s}^{e x p}$. To date, we are unable to understand if this is due to limitations of Eq.34 or poor quality of the available experimental data. Going into more details, we see that the deviations of our prediction, $\gamma_{t s}^{P W}$, from $\gamma_{t s}^{e x p}$ are limited for PS and within 5\% for OTP. Instead, the TS exponent of PMMA $\gamma_{t s}^{P W}$ is close to $\gamma_{t s}^{C R}$, but they both differ from the experimental value. All in all, since we cannot rule out that the anharmonicity parameters which are needed to evaluate $\gamma_{t s}$ from Eq.34 are affected by not 
negligible uncertainty, we conclude that additional work is needed to better validate Eq.34.

4.4.2. Elastic TS master curve We now derive the TS master curve of the structural relaxation. To this aim, we consider Eq.2 and Eq.3. Setting $\tau_{\alpha}\left(T_{g}\right)=100 \mathrm{~s}$, one finds $\widetilde{\Upsilon}_{0}=2-\widetilde{\Upsilon}_{1}-\widetilde{\Upsilon}_{2}=-11.70(1)$ [87]. Now, let us define

$$
Y=T \rho^{-\gamma_{t s}}
$$

Resorting to Eq.18 and Eq.34 recasts Eq.3 in terms of $Y$ as:

$$
X=\left(\frac{Y_{g}}{Y}\right)^{2 \delta_{L}+1}
$$

where $Y_{g}$ is the value of $Y$ at the glass transition. Plugging Eq.36 into Eq.2 one has

$$
\log \tau_{\alpha}=\widetilde{\Upsilon}_{0}^{\prime}+\widetilde{\Upsilon}_{1}\left(\frac{Y_{g}}{Y}\right)^{2 \delta_{L}+1}+\widetilde{\Upsilon}_{2}\left(\frac{Y_{g}}{Y}\right)^{2\left(2 \delta_{L}+1\right)}
$$

In Eq.37 rigorously $\widetilde{\Upsilon}_{0}^{\prime}=\widetilde{\Upsilon}_{0}$. However, we consider $\widetilde{\Upsilon}_{0}^{\prime}$ mildly adjustable in the range $\widetilde{\Upsilon}_{0}-0.5 \leq \widetilde{\Upsilon}_{0}^{\prime} \leq \widetilde{\Upsilon}_{0}+0.5$ to account for small errors in the determination of the glass transition. Taking $Y_{g}$ from the experiment, the total number of adjustable parameters of Eq.37 is two $\left(\widetilde{\Upsilon}_{0}^{\prime}\right.$ and the isochoric anharmonicity parameter $\left.\delta_{L}\right)$.

Notably, the shape of the master curve given by Eq.37 does not depend explicitly on the lattice Grüneisen parameter $\gamma_{L}$. Remind that, instead, the latter controls the change of the elastic modulus with the temperature under isobaric conditions, see Eq.19 and Eq.20.

The master curve Eq.37 allows to derive a compact expression of the isochoric fragility:

$$
m_{v}=\left.\frac{\partial \log \tau_{\alpha}}{\partial\left(Y_{g} / Y\right)}\right|_{Y_{g}}
$$

Plugging Eq.37 into Eq.38 leads to:

$$
m_{v}=\left(\widetilde{\Upsilon}_{1}+2 \widetilde{\Upsilon}_{2}\right)\left(2 \delta_{L}+1\right)
$$

Eq.39 establishes a linear relation between the isochoric fragility and the anharmonicity parameter $\delta_{L}$ where $\widetilde{\Upsilon}_{1}$ and $\widetilde{\Upsilon}_{2}$, the characteristic parameters of Eq.2, are regarded as universal quantities [87]. It is consistent with the known increase of the fragility with the anharmonicity $[51,52,53,54,55]$. This aspect becomes even clearer by considering the usual isobaric kinetic fragility:

$$
m=\left.\frac{\partial \log \tau_{\alpha}}{\partial\left(T_{g} / T\right)}\right|_{T_{g}} .
$$

A compact expression of the fragility $m$ is obtained by first deriving the temperature dependence the elastic modulus $G_{p}(T)$ from Eq.18 with $\rho(T)=\rho_{g} \exp \left[-\alpha\left(T-T_{g}\right)\right]$, plugging the expression into Eq.3, and the result into Eq.2. From the definition Eq.40 one has:

$$
m=\left(\widetilde{\Upsilon}_{1}+2 \widetilde{\Upsilon}_{2}\right)\left[1+2 \delta_{L}+\left(2 \gamma_{L}+\frac{1}{3}\right) \alpha T_{g}\right]
$$




\begin{tabular}{|c|c|c|c|c|c|c|c|}
\hline & System & $\gamma_{t s}$ & $\delta_{L}$ & $\widetilde{\Upsilon_{0}^{\prime}}$ & $m_{v}^{f i t}$ & $m_{v}^{e x p}$ & Ref. \\
\hline 1 & PCB62 & 8.5 & 0.103 & -11.35 & $29 \pm 4$ & $24 \pm 1$ & [138] \\
\hline 2 & BMPC & 7.0 & 0.095 & -11.58 & $29 \pm 4$ & $25 \pm 1$ & {$[139,140]$} \\
\hline 3 & BMMPC & 8.5 & 0.052 & -11.65 & $26 \pm 5$ & $26 \pm 1$ & [139] \\
\hline 4 & PCB54 & 6.7 & 0.204 & -11.78 & $34 \pm 5$ & $31 \pm 3$ & [138] \\
\hline 5 & PCB42 & 5.5 & 0.381 & -11.40 & $42 \pm 5$ & $35 \pm 5$ & [138] \\
\hline 6 & vitamin $\mathrm{E}$ & 3.9 & 0.127 & -12.20 & $30 \pm 5$ & $36 \pm 6$ & [141] \\
\hline 7 & $\mathrm{KDE}$ & 4.5 & 0.319 & -11.21 & $39 \pm 6$ & $39 \pm 3$ & [142] \\
\hline 8 & salol & 5.2 & 0.520 & -11.20 & $49 \pm 5$ & $40 \pm 5$ & [143] \\
\hline 9 & PCHMA & 2.9 & 0.120 & -12.20 & $30 \pm 5$ & $42 \pm 8$ & [144] \\
\hline 10 & PMMA-3 & 3.7 & 0.308 & -11.93 & $39 \pm 5$ & $43 \pm 2$ & [145] \\
\hline 11 & PDE & 4.4 & 0.409 & -11.67 & $44 \pm 5$ & $45 \pm 4$ & {$[139,146,142]$} \\
\hline 12 & DC704 & 6.15 & 0.542 & -11.50 & $50 \pm 5$ & $47 \pm 5$ & [147] \\
\hline 13 & PMMA-4 & 3.2 & 0.308 & -12.20 & $39 \pm 5$ & $49 \pm 3$ & [145] \\
\hline 14 & POB & 2.65 & 0.368 & -12.20 & $42 \pm 7$ & $50 \pm 6$ & [148] \\
\hline 15 & $1,4 \mathrm{PI}$ & 3.5 & 0.406 & -12.19 & $43 \pm 6$ & $51 \pm 7$ & {$[149,150,5]$} \\
\hline 16 & PVAc & 2.6 & 0.486 & -11.20 & $47 \pm 8$ & $52 \pm 5$ & {$[151]$} \\
\hline 17 & PMPS & 5.63 & 0.623 & -11.77 & $54 \pm 5$ & $54 \pm 3$ & {$[152]$} \\
\hline 18 & OTP & 4.0 & 0.585 & -11.75 & $52 \pm 5$ & $54 \pm 2$ & {$[153,154,155,156,157,158]$} \\
\hline 19 & verapamil $\mathrm{HCl}$ & 2.47 & 0.564 & -11.87 & $51 \pm 4$ & $57 \pm 3$ & {$[159,160]$} \\
\hline 20 & DGEBA & 2.8 & 0.909 & -11.85 & $60 \pm 6$ & $57 \pm 7$ & {$[161]$} \\
\hline 21 & DPVC & 3.2 & 0.904 & -11.20 & $67 \pm 5$ & $62 \pm 3$ & {$[162]$} \\
\hline 22 & PMTS & 5.0 & 0.500 & -12.10 & $48 \pm 5$ & $63 \pm 2$ & {$[163]$} \\
\hline 23 & PCGE & 3.3 & 0.808 & -11.20 & $63 \pm 6$ & $63 \pm 3$ & [164] \\
\hline 24 & $1,4 \mathrm{~PB}$ & 1.8 & 0.480 & -12.02 & $47 \pm 12$ & $64 \pm 6$ & [4] \\
\hline 25 & PPGE & 3.45 & 0.734 & -11.84 & $59 \pm 5$ & $65 \pm 4$ & {$[165,166]$} \\
\hline 26 & $\mathrm{PC}$ & 3.8 & 0.792 & -11.20 & $62 \pm 7$ & $66 \pm 4$ & [167] \\
\hline 27 & PVME & 2.5 & 0.472 & -12.23 & $47 \pm 6$ & $66 \pm 7$ & [168] \\
\hline 28 & PMMA-20 & 1.94 & 0.561 & -12.14 & $51 \pm 6$ & $67 \pm 13$ & [134] \\
\hline 29 & DGEBA-epon & 3.5 & 0.909 & -11.85 & $68 \pm 8$ & $70 \pm 8$ & [169] \\
\hline 30 & PPG4000 & 2.5 & 0.745 & -12.20 & $60 \pm 7$ & $76 \pm 15$ & {$[170]$} \\
\hline 31 & PMMA-10 & 1.8 & 0.766 & -12.20 & $61 \pm 12$ & $85 \pm 20$ & [145] \\
\hline 32 & $1,2 \mathrm{~PB}$ & 1.89 & 0.592 & -12.20 & $52 \pm 8$ & $86 \pm 15$ & [142] \\
\hline 33 & PS & 2.27 & 1.37 & -11.77 & $90 \pm 12$ & $104 \pm 8$ & [171] \\
\hline 34 & sorbitol & 0.18 & 1.35 & -12.18 & $89 \pm 9$ & $112 \pm 10$ & {$[172,2,173]$} \\
\hline
\end{tabular}

Table 2. Best-fit values of the parameters of the TS master curve Eq.37 ( $\delta_{L}$ and $\widetilde{\Upsilon}_{0}^{\prime}$, adjusted in the range $\widetilde{\Upsilon}_{0}-0.5 \leq \widetilde{\Upsilon}_{0}^{\prime} \leq \widetilde{\Upsilon}_{0}+0.5$ with $\widetilde{\Upsilon}_{0}=-11.70(1)$ [87]) for the glassformers in Fig.7, Fig.8, Fig.9. The experimental characteristic exponent $\gamma_{t s}$, the experimental isochoric fragility $m_{v}^{e x p}$, Eq.38, and the best-fit value $m_{v}^{f i t}$, evaluated via Eq.39, are also listed. The glassformers are listed in increasing order of the isochoric fragility $m_{v}^{e x p}$. 

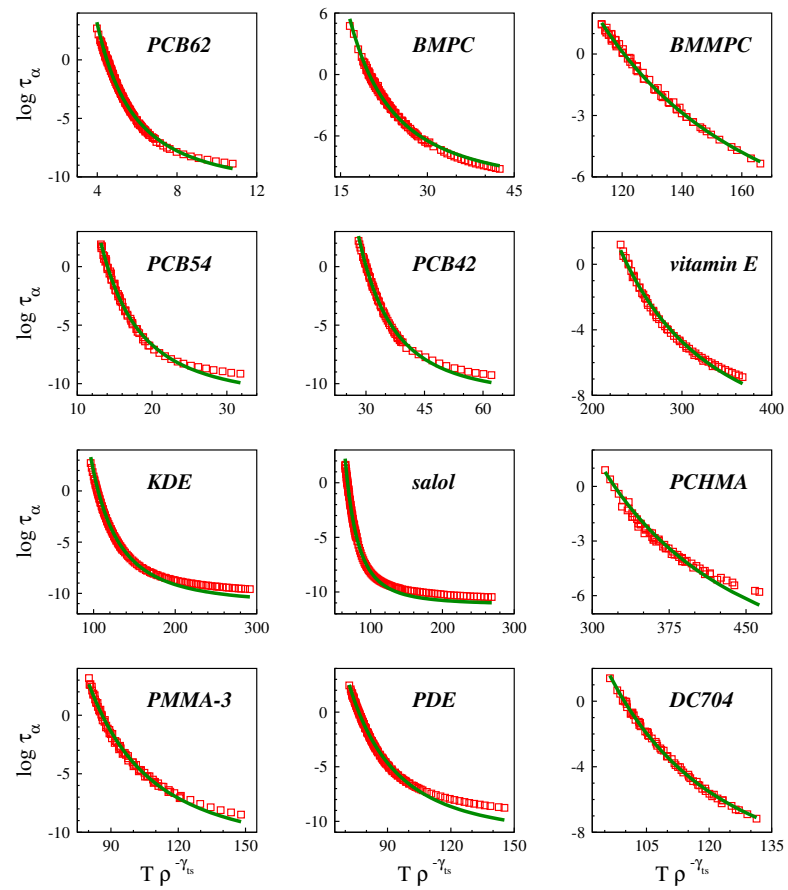

Figure 7. TS master curve $\log \tau_{\alpha}$ vs $T \rho^{-\gamma_{t s}}$ of selected glassformers (squares) with lower isochoric fragility. The best-fit with Eq.37 is superimposed (continuous line). Best-fit values in Table 2.

Eq.41 relates the kinetic fragility to parameters all related to the anharmonicity and predicts $m \geq m_{\min }=\widetilde{\Upsilon}_{1}+2 \widetilde{\Upsilon}_{2}=24 \pm 1.6$, assuming $\delta_{L}=\alpha=0$ for harmonic liquids. By the way, by replacing Eq.34 into Eq.41, the isobaric fragility reduces to the expression derived by Casalini and Roland [142]:

$$
m=m_{v}\left[1+\gamma_{t s} \alpha T_{g}\right]
$$

Fig.7, Fig.8, and Fig.9 show the comparison of the theoretical TS master curve of the structural relaxation, Eq.37, with TS of thirty-four different glassformers, including van der Waals liquids and polymers in a wide range of isochoric fragilities $\left(24 \leq m_{v} \leq 112\right)$. The best-fit parameters $\left(\delta_{L}\right.$ and $\widetilde{\Upsilon}_{0}^{\prime}$, adjusted in the range $\left.\widetilde{\Upsilon}_{0}-0.5 \leq \widetilde{\Upsilon}_{0}^{\prime} \leq \widetilde{\Upsilon}_{0}+0.5\right)$ are listed in Table 2. The quality of the fit provided by Eq.37 - having two adjustable parameters - is generally good with nice agreement around the glass transition. This is interpreted by noting that Eq. 37 stems from Eq. 2 which covers the GT region too [87]. Some disagreement between the experimental and the theoretical TS master curves occurs for short relaxation times. The accuracy of Eq.37 is found to be comparable to the one of ref.[13] with the same number of adjustable parameters. Fig. 10 plots the distribution of the best-fit values of the anharmonicity parameters $\delta_{L}$. The distribution peaks around 0.5 and, apart from two exceptions, does not exceed the unit value. Interestingly, a number of glassformers approaches the harmonic value $\delta_{L}=0$.

Fig.11 correlates the experimental isochoric fragilities $m_{v}^{\text {exp }}$ and the isochoric anharmonicity parameter $\delta_{L}$, as drawn by the best-fit of Eq.37 to the experimental TS master 

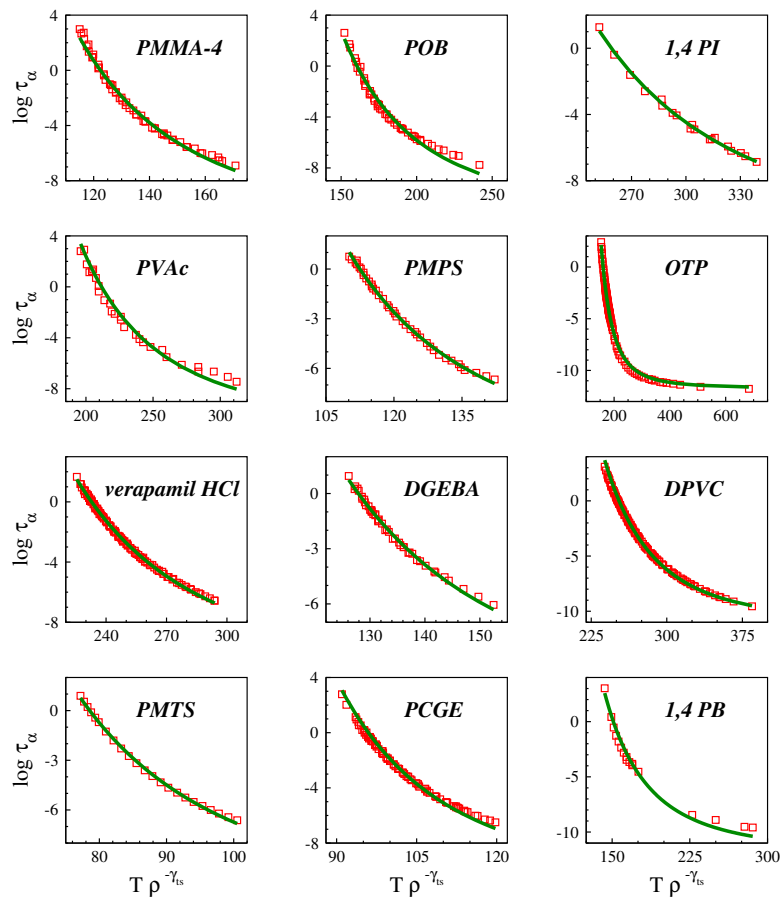

Figure 8. TS master curve $\log \tau_{\alpha}$ vs $T \rho^{-\gamma_{t s}}$ of selected glassformers (squares) with intermediate isochoric fragility. Other details as in Fig.7.
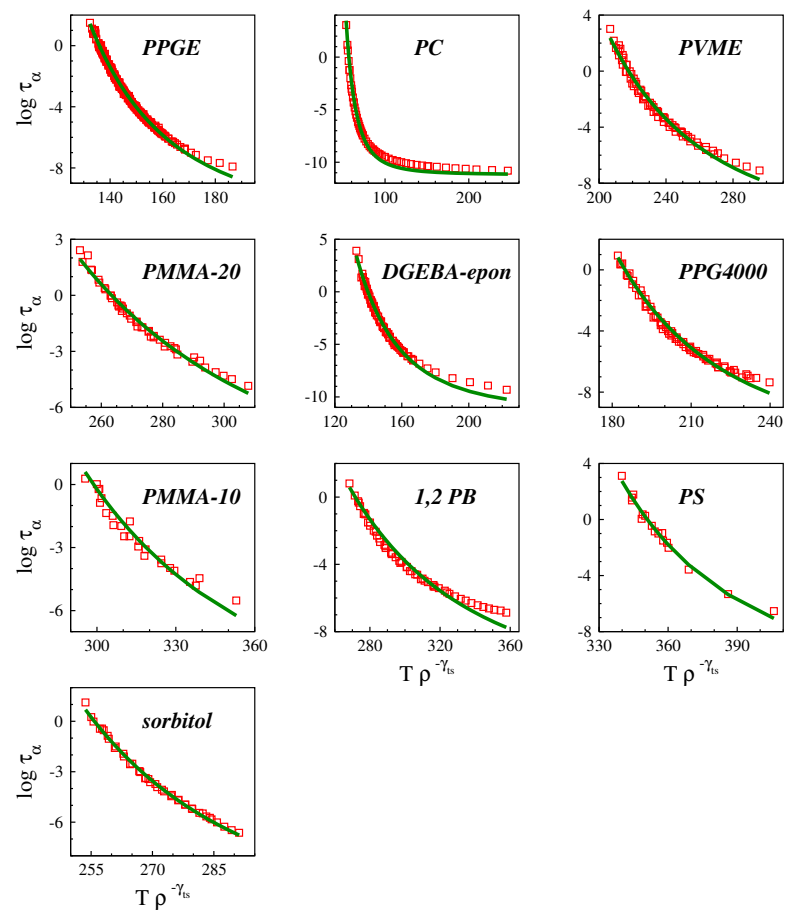

Figure 9. TS master curve $\log \tau_{\alpha}$ vs $T \rho^{-\gamma_{t s}}$ of selected glassformers (squares) with higher isochoric fragility. Other details as in Fig.7. 


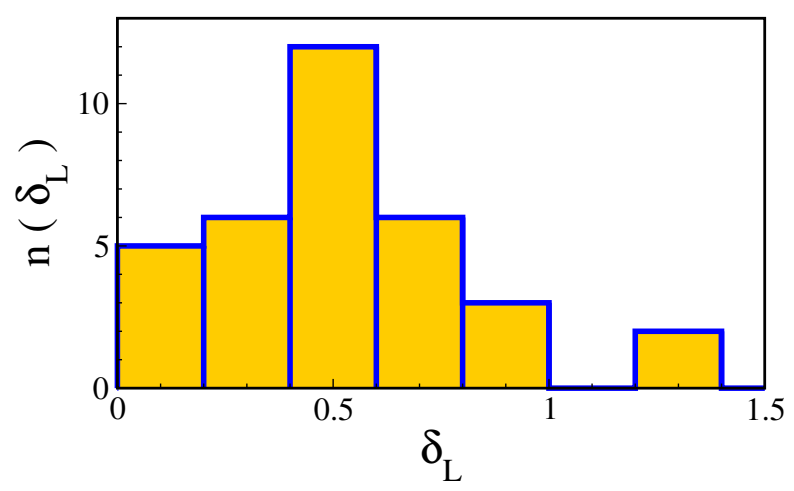

Figure 10. Distribution of the best-fit values of the isochoric anharmonicity parameter $\delta_{L}$ as drawn by TS of the structural relaxation, see Table 2 .

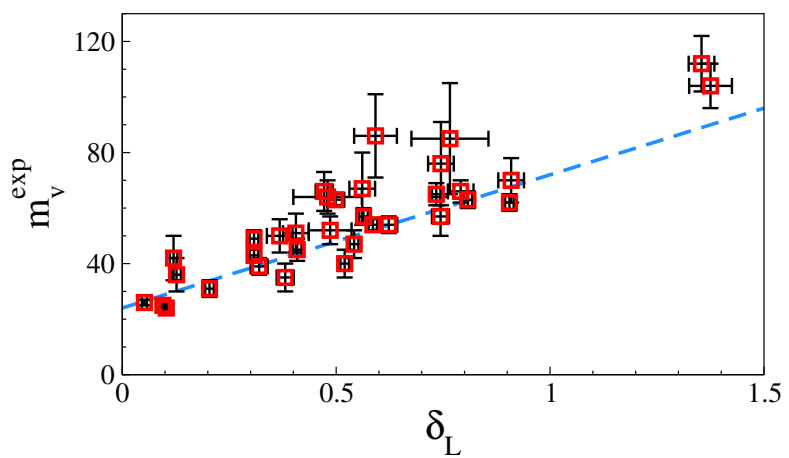

Figure 11. Correlation plot between the experimental isochoric fragilities of the thirty-four different glassformers examined, and the corresponding isochoric anharmonicity parameter $\delta_{L}$, as drawn by the best-fit of Eq.37 to the experimental TS master curves (Pearson correlation coefficient $R=0.89$ ). See Table 2 for numerical values. The dashed line is Eq.39 with no adjustable parameters and $\widetilde{\Upsilon}_{1}=3.4(2), \widetilde{\Upsilon}_{2}=10.3(8)$ [87].

curves, see Table 2. The correlation is good (Pearson correlation coefficient $R=0.89$ ) and in good agreement with Eq.39 which has no adjustable parameters. We see that the isochoric fragility increases with the anharmonicity, as accounted for by the isochoric anharmonicity parameter $\delta_{L}$. The finding that the anharmonicity enhances the fragility parallels many other similar conclusions $[51,52,53,54,55]$.

To proceed, we compare in Table 3 the anharmonicity parameters $\delta_{L}$, as drawn by thermodynamics, see Table 1, and from TS, see Table 2. The comparison is limited to PMMA, PS and OTP. We always find $\delta_{L}^{t d}<\delta_{L}^{t s}$ with better agreement for PMMA.

4.4.3. Vibrational isochoric heat capacity Anharmonicty affects the heat capacity, see Sec.2.3. Here, we compare the vibrational isochoric heat capacity as predicted by the present work, Eq. 16, and the alternative approach of Ref. [119] leading to Eq.17 where $T=T_{g}$ is set. Eq. 16 and Eq. 17 predict that the relative change of $C_{V}^{v i b}$ due to the anharmonicity is $\delta_{L}$ and $\alpha T_{g}(q-1)$, respectively. Since $q \sim 2$ [119], we compare $\delta_{L}$ and $\alpha T_{g}$ in Table 3 for 
Table 3. Anharmonicity parameter from thermodynamics, $\delta_{L}^{t d}$, see Table 1, and from TS of the structural relaxation, $\delta_{L}^{t s}$, see Table 2 . The quantity $\alpha T_{g}$ at ambient pressure is also listed with data from the references listed in Table 2, apart from PMMA [121] and PVAc, OTP and PS [117].

\begin{tabular}{llll}
\hline \hline & $\delta_{L}^{t d}$ & $\delta_{L}^{t s}$ & $\alpha T_{g}$ \\
\hline PCB62 & & 0.103 & 0.19 \\
vitamin E & & 0.127 & 0.13 \\
PCB54 & & 0.204 & 0.15 \\
PCB42 & & 0.381 & 0.15 \\
PVAc & & 0.486 & 0.22 \\
OTP & 0.32 & 0.585 & 0.17 \\
PMMA & 0.67 & 0.766 & 0.19 \\
PS & 0.92 & 1.37 & 0.21 \\
\hline \hline
\end{tabular}

systems with different isochoric fragility $m_{v}$. They are relatively close for systems with low isochoric fragility, e.g. vitamin E and PCB54. However, considering a larger range of $m_{v}$, it is seen that the quantity $\alpha T_{g}$ changes mildly, less than about $60 \%$, whereas $\delta_{L}$ exhibits much stronger variation. The nearly constant value of $\alpha T_{g}$ is well known in polymer physics where the "quasi-universal" value $\alpha T_{g}=0.164$ is reported [121]. The above analysis suggests that, in order to discriminate between the predictions of Eq. 16 and Eq.17, one has to consider glassformers with high isochoric fragility. We are aware that further analysis, beyond the purpose of the present paper, is needed to better assess Eq. 16 and Eq.17. Nonetheless, we cannot refrain from one remark. By combining Eq.16 and Eq.39, one establishes a relation between the isochoric fragility $m_{v}$ and the increase $\Delta C_{V}^{v i b}$ with respect to the harmonic value due to the presence of anharmonicity:

$$
\Delta C_{V}^{v i b}=\frac{3 N k_{B}}{2}\left[\frac{m_{v}}{m_{v}^{\min }}-1\right]
$$

with $m_{v}^{\text {min }}=\widetilde{\Upsilon}_{1}+2 \widetilde{\Upsilon}_{2}$ the minimum isochoric fragility for harmonic liquids. It is tempting to note that Eq.43 is reminiscent of the well-known correlation between the kinetic fragility and the size of the jump of the isobaric thermal capacity observed by traversing the glass transition from below, a process leading to anharmonicity increase [174].

\section{Conclusions}

The present paper presents a scheme to evaluate both the TS characteristic exponent $\gamma_{t s}$ and the master curve by resorting to experimentally accessible quantities characterising the anharmonic elasticity and no details about the microscopic interactions. Two central parameters are identified, i.e. the lattice Grüneisen parameter $\gamma_{L}$ and the isochoric anharmonicity $\delta_{L}$. Our analysis suggests that their variations are rather limited across states of 
the TS region, thus setting the characteristic TS exponent $\gamma_{t s}$ and the shape of the TS master curve.

We express the exponent $\gamma_{t s}$ in terms of $\gamma_{L}$ and $\delta_{L}$. We also find an analytic expression of the TS master curve of the structural relaxation where the adjustable parameter is the isochoric anharmonicity $\delta_{L}$ together with a narrowly varied vertical shift. The comparison with the experimental TS master curves of thirty-four glassformers, including van der Waals liquids and polymers, is convincing and good agreement is generally seen close to the glass transition where elastic response is well developed. We tested against the available experimental data concerning three glassformers - two polymers (PMMA, PS) and one molecular liquid (OTP) -: i) our prediction of the characteristic exponent $\gamma_{t s}$ in terms of parameters characterizing the anharmonic elasticity, ii) the isochoric anharmonicity $\delta_{L}$, as drawn by the best-fit of TS of the structural relaxation. The results are encouraging, but they suffer from the scarcity and the uncertainty of data concerning the anharmonic elasticity in the liquid state.

The elastic interpretation of TS predicts a linear relation between the isochoric fragility and the isochoric anharmonicity with no adjustable parameters. It agrees rather favourably with the experimental data under consideration. A related expression concerning the usual isobaric fragility $m$ is presented. It predicts $m \geq 24 \pm 1.6$.

As side outcome of our treatment of the anharmonic elasticity, a relation between the increase of the isochoric vibrational heat capacity due to anharmonicity and the isochoric fragility is derived.

\section{Acknowledgments}

S. Capaccioli is warmly thanked for helpful discussions and the critical reading of the manuscript. A generous grant of computing time from IT Center, University of Pisa and Dell ${ }^{\mathrm{B}}$ Italia is gratefully acknowledged. 
[1] A. G. S. Hollander and K. O. Prins. Atactic polypropylene at high pressure. i. deuteron-nmr determination of the glass-transition temperature. J. Non-Cryst. Solids, 286:1-11, 2001.

[2] R. Casalini and C. M. Roland. Thermodynamical scaling of the glass transition dynamics. Phys. Rev. E, 69:062501, 2004.

[3] C. Dreyfus, A. Le Grand, J. Gapinski, W. Steffen, and A. Patkowski. Scaling the $\alpha$-relaxation time of supercooled fragile organic liquids. Eur. Phys. J. B, 42:309-319, 2004.

[4] C. Alba-Simionesco, A. Cailliaux, A. Alegría, and G. Tarjus. Scaling out the density dependence of the relaxation in glass-forming polymers. EPL, 68:58, 2004.

[5] C. M. Roland, S. Hensel-Bielowka, M. Paluch, and R. Casalini. Supercooled dynamics of glass-forming liquids and polymers under hydrostatic pressure. Rep. Prog. Phys., 68:1405, 2005.

[6] C. M. Roland. Relaxation phenomena in vitrifying polymers and molecular liquids. Macromolecules, 43:7875-7890, 2010.

[7] R. Casalini and C. M. Roland. Determination of the thermodynamic scaling exponent for relaxation in liquids from static ambient-pressure quantities. Phys. Rev. Lett., 113:085701, 2014.

[8] Ludovic Berthier and Giulio Biroli. Theoretical perspective on the glass transition and amorphous materials. Rev. Mod. Phys., 83:587-645, 2011.

[9] M. D. Ediger and P. Harrowell. Perspective: Supercooled liquids and glasses. J. Chem. Phys., 137:080901, 2012.

[10] M. C. C. Ribeiro, T. Scopigno, and G. Ruocco. Computer simulation study of thermodynamic scaling of dynamics of $2 \mathrm{ca}\left(\mathrm{no}_{3}\right)_{2} \cdot 3 \mathrm{kno}_{3}$. J. Chem. Phys., 135:164510, 2011.

[11] O. Chulkin. Microscopic Dynamics of Polymer Melts: Numerical Simulations with Coarse-Grained Models. PhD thesis, Graduate School of Basic Sciences "Galileo Galilei" - Applied Physics, Universita' di Pisa, 2011.

[12] F. Puosi, C. De Michele, and D. Leporini. Scaling between relaxation, transport and caged dynamics in a binary mixture on a per-component basis. J. Chem. Phys., 138:12A532, 2013.

[13] F. Puosi, O. Chulkin, S. Bernini, S. Capaccioli, and D. Leporini. Thermodynamic scaling of vibrational dynamics and relaxation. J. Chem. Phys., 145:234904, 2016.

[14] C. M. Roland, S. Bair, and R. Casalini. Thermodynamic scaling of the viscosity of van der waals, hbonded, and ionic liquids. J. Chem. Phys., 125:124508, 2006.

[15] E. R. Lopez, A. S. Pensado, M. J. P. Comunas, A. A. H. Padua, J. Fernandez, and K. R. Harris. Density scaling of the transport properties of molecular and ionic liquids. J. Chem. Phys., 134:144507, 2011.

[16] M. Paluch, S. Haracz, A. Grzybowski, M. Mierzwa, J. Pionteck, A. Rivera-Calzada, and C. Leon. A relationship between intermolecular potential, thermodynamics, and dynamic scaling for a supercooled ionic liquid. J. Phys. Chem. Lett., 1:987-992, 2010.

[17] S. Urban and C. M. Roland. Low frequency relaxation in liquid crystals in relation to structural relaxation in glass-formers. J. Non-Cryst. Solids, 357:740-745, 2011.

[18] M. Romanini, M. Barrio, S. Capaccioli, R. Macovez, M. D. Ruiz-Martin, and J. Ll. Tamarit. Double primary relaxation in a highly anisotropic orientational glass-former with low-dimensional disorder. $J$. Phys. Chem. C, 120:10614-10621, 2016.

[19] C. M. Roland, R. Casalini, R. Bergman, and J. Mattsson. Role of hydrogen bonds in the supercooled dynamics of glass-forming liquids at high pressures. Phys. Rev. B, 77:012201, 2008.

[20] W. G. Hoover and M. Rossj. Statistical theories of melting. Contemporary Physics, 12:339-356, 1971.

[21] D. Coslovich and C. M. Roland. Thermodynamic scaling of diffusion in supercooled lennard-jones liquids. J. Phys. Chem. B, 112:1329-1332, 2008.

[22] N. Gnan, T. B. Schrøder, U. R. Pedersen, N. P. Bailey, and J. C. Dyre. Pressure-energy correlations in liquids. iv. "isomorphs" in liquid phase diagrams. J. Chem. Phys., 131:234504, 2009.

[23] T. B. Schrøder, U. R. Pedersen, N. P. Bailey, S. Toxvaerd, and J. C. Dyre. Hidden scale invariance in molecular van der waals liquids: A simulation study. Phys. Rev. E, 80:041502, 2009.

[24] J. C. Dyre. Hidden scale invariance in condensed matter. J.Phys. Chem. B, 118:10007-10024, 2014.

[25] Yuan-Chao Hu, Bao-Shuang Shang, Peng-Fei Guan, Yong Yang, Hai-Yang Bai, and Wei-Hua Wang. Thermodynamic scaling of glassy dynamics and dynamic heterogeneities in metallic glass-forming 
liquid. J. Chem.Phys., 145(10):104503, 2016.

[26] N. P. Bailey, U. R. Pedersen, N. Gnan, T. B. Schrøder, and J. C. Dyre. Pressure-energy correlations in liquids. i. results from computer simulations. J. Chem. Phys., 129:184507, 2008.

[27] D. Coslovich and C.M. Roland. Heterogeneous slow dynamics and the interaction potential of glassforming liquids. J. Non-Cryst. Solids, 357:397-400, 2011.

[28] F. Puosi. Relaxation, short time dynamics and elastic properties in glass-forming liquids. PhD thesis, Graduate School of Basic Sciences "Galileo Galilei" - Applied Physics, Universita' di Pisa, (https://etd.adm.unipi.it/theses/available/etd-05182012-171519/), 2012.

[29] Arno A. Veldhorst, Jeppe C. Dyre, and Thomas B. Schrøder. Scaling of the dynamics of flexible lennardjones chains: Effects of harmonic bonds. J. Chem. Phys., 143:194503, 2015.

[30] G. Tarjus, D. Kivelson, S. Mossa, and C. Alba-Simionesco. Disentangling density and temperature effects in the viscous slowing down of glassforming liquids. J. Chem. Phys., 120:6135-6141, 2004.

[31] R. Casalini, U. Mohanty, and C. M. Roland. Thermodynamic interpretation of the scaling of the dynamics of supercooled liquids. J. Chem. Phys., 125:014505, 2006.

[32] R. Casalini and C.M. Roland. An equation for the description of volume and temperature dependences of the dynamics of supercooled liquids and polymer melts. J. Non-Cryst. Solids, 353:3936 - 3939, 2007.

[33] E. Masiewicz, A. Grzybowski, A. P. Sokolov, and M. Paluch. Temperature-volume entropic model for viscosities and structural relaxation times of glass formers. J. Phys. Chem. Lett., 3:2643-2648, 2012.

[34] I. Avramov. Viscosity in disordered media. J. Non-Cryst. Solids, 351:3163 - 3173, 2005.

[35] J. C. Mauro, Y. Z. Yue, A. J. Ellison, P. K. Gupta, and D. C.Allan. Viscosity of glass-forming liquids. Proc. Natl. Acad. Sci. USA, 106:19780-19784, 2009.

[36] J. D. Ferry. Viscoelastic Properties of Polymers. Wiley, New York, 1980.

[37] F. Puosi and D. Leporini. Correlation of the instantaneous and the intermediate-time elasticity with the structural relaxation in glassforming systems. J. Chem. Phys., 136:041104, 2012.

[38] A. V. Tobolsky. Properties and Structure of Polymers. Wiley, New York, 1960.

[39] S. Bernini and D. Leporini. Short-time elasticity of polymer melts: Tobolsky conjecture and heterogeneous local stiffness. J.Pol.Sci., Part B: Polym. Phys., 53:1401-1407, 2015.

[40] J. Clerk Maxwell. On the dynamical theory of gases. Phil. Trans. R. Soc. Lond., 157:49-88, 1867.

[41] J. Frenkel. über die wärmebewegung in festen und flüssigen körpern. Z. für Phys., 35:652-669, 1926.

[42] J. Frenkel. Continuity of the solid and the liquid states. Nature, 136:167-168, 1935.

[43] J. Frenkel. Kinetic Theory of Liquids. Dover Publications, New York, 1955.

[44] K. Trachenko and V. V. Brazhkin. Collective modes and thermodynamics of the liquid state. Rep. Prog. Phys., 79:016502, 2016.

[45] A. Tobolsky, R. E. Powell, and H. Eyring. Elastic-viscous properties of matter. In R. E. Burk and O. Grummit, editors, Frontiers in Chemistry, volume 1, pages 125-190, New York, 1943. Interscience.

[46] T. H. K. Barron, J. G. Collins, and G. K. White. Thermal expansion of solids at low temperatures. Adv. Phys., 29:609-730, 1980.

[47] T. H. K. Barron and G. K. White. Heat Capacity and Thermal Expansion at Low Temperatures. Springer, New York, 1999.

[48] T. H. K. Barron. Gruneisen parameters for the equation of state of solids. Annals of Physics, 1:77-90, 1957.

[49] O. L. Anderson. Equations of State of Solids for Geophysics and Ceramic Science. Oxford University Press, Oxford, 1995.

[50] A. R. Oganov. Thermodynamics, phase transitions, equations of state, and elasticity of minerals at high pressures and temperatures. In Gerald Schubert, editor, Treatise on Geophysics, volume 2, pages 179202. Elsevier, Amsterdam, ii edition, 2015.

[51] C. A. Angell. Formation of glasses from liquids and biopolymers. Science, 267:1924-1935, 1995.

[52] A. P. Sokolov. Why the glass transition is still interesting. Science, 273:1675-1676, 1996.

[53] V. N. Novikov. Vibration anharmonicity and fast relaxation in the region of the glass transition. Phys. Rev. B, 58:8367-8378, 1998.

[54] P. Bordat, F. Affouard, M. Descamps, and K. L. Ngai. Does the interaction potential determine both the 
fragility of a liquid and the vibrational properties of its glassy state? Phys. Rev. Lett., 93:105502, 2004.

[55] V. N. Novikov, Y. Ding, and A. P. Sokolov. Correlation of fragility of supercooled liquids with elastic properties of glasses. Phys.Rev.E, 71:061501, 2005.

[56] K. L. Ngai. Dynamic and thermodynamic properties of glass-forming substances. J. Non-Cryst. Solids, 275:7-51, 2000.

[57] K. L. Ngai. Short-time and long-time relaxation dynamics of glass-forming substances: a coupling model perspective. J. Phys.: Condens. Matter, 12:6437-6451, 2000.

[58] K. L. Ngai. Relaxation and Diffusion in Complex Systems. Springer, Berlin, 2011.

[59] C.M. Roland, J.L. Feldman, and R. Casalini. Scaling of the local dynamics and the intermolecular potential. J. Non-Cryst. Solids, 352:4895-4899, 2006.

[60] T. B. Schrøder, N. P. Bailey, U. R. Pedersen, N. Gnan, and J. C. Dyre. Pressure-energy correlations in liquids. iii. statistical mechanics and thermodynamics of liquids with hidden scale invariance. J. Chem. Phys., 131:234503, 2009.

[61] A. Widmer-Cooper, HEIDI Perry, PETER Harrowell, and DAVID R. Reichman. Irreversible reorganization in a supercooled liquid originates from localized soft modes. Nature Physics, 4:711$715,2008$.

[62] Asaph Widmer-Cooper, Heidi Perry, Peter Harrowell, and David R. Reichman. Localized soft modes and the supercooled liquid's irreversible passage through its configuration space. J.Chem.Phys., 131:194508, 2009.

[63] M. Wyart. Correlations between vibrational entropy and dynamics in liquids. Phys. Rev. Lett., 104:095901, 2010.

[64] F. Puosi and D. Leporini. Spatial displacement correlations in polymeric systems. J. Chem. Phys., 136:164901, 2012.

[65] F. Puosi and D. Leporini. Erratum: "spatial displacement correlations in polymeric systems" [j. chem. phys.136, 164901 (2012)]. J. Chem. Phys., 139:029901, 2013.

[66] Smarajit Karmakar, Chandan Dasgupta, and Srikanth Sastry. Short-time beta relaxation in glass-forming liquids is cooperative in nature. Phys. Rev. Lett., 116:085701, 2016.

[67] S. Bernini and D. Leporini. Cage effect in supercooled molecular liquids: Local anisotropies and collective solid-like response. J. Chem. Phys., 144:144505, 2016.

[68] Ooshida Takeshi, Susumu Goto, Takeshi Matsumoto, and Michio Otsuki. Calculation of displacement correlation tensor indicating vortical cooperative motion in two-dimensional colloidal liquids. Phys. Rev. E, 94:022125, 2016.

[69] S. Bernini, F. Puosi, M. Barucco, and D. Leporini. Competition of the connectivity with the local and the global order in polymer melts and crystals. J. Chem. Phys., 139:184501, 2013.

[70] S. Bernini and D. Leporini. Anisotropy of the monomer random walk in a polymer melt: local-order and connectivity effects. J. Phys.: Condens. Matter, 28:185103, 2016.

[71] S. Bernini, F. Puosi, and D. Leporini. Weak links between fast mobility and local structure in molecular and atomic liquids. J. Chem. Phys., 142:124504, 2015.

[72] S. Bernini, F. Puosi, and D. Leporini. Cage rattling does not correlate with the local geometry in molecular liquids. J. Non-Cryst. Solids, 407:29-33, 2015.

[73] J. C. Dyre. The glass transition and elastic models of glass-forming liquids. Rev. Mod. Phys., 78:953-972, 2006.

[74] S.V. Nemilov. Interrelation between shear modulus and the molecular parameters of viscous flow for glass forming liquids. J. Non-Cryst. Sol., 352:2715-2725, 2006.

[75] A. Lemaitre. Structural relaxation is a scale-free process. Phys. Rev. Lett., 113:245702, 2014.

[76] A.V. Granato. The specific heat of simple liquids. J. Non-Cryst. Solids, 307-310:376-386, 2002.

[77] L. Yan, G. Düring, and M. Wyart. Why glass elasticity affects the thermodynamics and fragility of supercooled liquids. PNAS, 110:6307-6312, 2013.

[78] V. N. Novikov and A. P. Sokolov. Poisson's ratio and the fragility of glass-forming liquids. Nature, 431:961-963, 2004.

[79] J. C. Dyre and W. H. Wang. The instantaneous shear modulus in the shoving model. J.Chem.Phys., 
136:224108, 2012.

[80] J. P. Wittmer, H. Xu, P. Polińska, F. Weysser, and J. Baschnagel. Shear modulus of simulated glassforming model systems: Effects of boundary condition, temperature, and sampling time. J. Chem. Phys., 138:12A533, 2013.

[81] Stephen Mirigian and Kenneth S. Schweizer. Elastically cooperative activated barrier hopping theory of relaxation in viscous fluids.i. general formulation and application to hard sphere fluids. J. Chem. Phys., 140:194506, 2014.

[82] Stephen Mirigian and Kenneth S. Schweizer. Elastically cooperative activated barrier hopping theory of relaxation in viscous fluids. ii. thermal liquids. J. Chem. Phys., 140:194507, 2014.

[83] Beatriz A. Pazmiño Betancourt, Paul Z. Hanakata, Francis W. Starr, and Jack F. Douglas. Quantitative relations between cooperative motion, emergent elasticity, and free volume in model glass-forming polymer materials. Proc. Natl. Acad. Sci. USA, 112:2966-2971, 2015.

[84] Jack F. Douglas, Beatriz A. Pazmiño Betancourt, Xuhang Tong, and Hao Zhang. Localization model description of diffusion and structural relaxation in glass-forming cu-zr alloys. J. Stat. Mech.: Theory Exp., page 054048, 2016.

[85] Alessio Zaccone and Eugene M. Terentjev. Disorder-assisted melting and the glass transition in amorphous solids. Phys. Rev. Lett., 110:178002, 2013.

[86] Johannes Krausser, Konrad H. Samwer, and Alessio Zaccone. Interatomic repulsion softness directly controls the fragility of supercooled metallic melts. Proc. Natl. Acad. Sci. USA, 112:13762-13767, 2015.

[87] F. Puosi and D. Leporini. The kinetic fragility of liquids as manifestation of the elastic softening. Eur. Phys. J. E, 38:87, 2015.

[88] Shibu Saw and Peter Harrowell. Rigidity in condensed matter and its origin in configurational constraint. Phys. Rev. Lett., 116:137801, 2016.

[89] L. Larini, A. Ottochian, C. De Michele, and D. Leporini. Universal scaling between structural relaxation and vibrational dynamics in glass-forming liquids and polymers. Nature Physics, 4:42-45, 2008.

[90] A. Ottochian, C. De Michele, and D. Leporini. Universal divergenceless scaling between structural relaxation and caged dynamics in glass-forming systems. J. Chem. Phys., 131:224517, 2009.

[91] A. Ottochian and D. Leporini. Universal scaling between structural relaxation and caged dynamics in glass-forming systems: Free volume and time scales. J. Non-Cryst. Solids, 357:298-301, 2011.

[92] A. Ottochian and D. Leporini. Scaling between structural relaxation and caged dynamics in $c a_{0.4} k_{0.6}\left(\mathrm{no}_{3}\right)_{1.4}$ and glycerol: free volume, time scales and implications for the pressure-energy correlations. Philosophical Magazine, 91:1786-1795, 2011.

[93] C. De Michele, E. Del Gado, and D. Leporini. Scaling between structural relaxation and particle caging in a model colloidal gel. Soft Matter, 7:4025-4031, 2011.

[94] F. Puosi and D. Leporini. Scaling between relaxation, transport, and caged dynamics in polymers: From cage restructuring to diffusion. J.Phys. Chem. B, 115:14046-14051, 2011.

[95] F. Puosi and D. Leporini. Communication: Fast and local predictors of the violation of the stokes- einstein law in polymers and supercooled liquids. J. Chem. Phys., 136:211101, 2012.

[96] A. Ottochian, F. Puosi, C. De Michele, and D. Leporini. Comment on "generalized localization model of relaxation in glass-forming liquids". Soft Matter, 9:7890-7891, 2013.

[97] V. N. Novikov and A. P. Sokolov. Role of quantum effects in the glass transition. Phys. Rev. Lett., 110:065701, 2013.

[98] A. Barbieri, G. Gorini, and D. Leporini. Role of the density in the crossover region of o-terphenyl and poly(vinyl acetate). Phys. Rev. E, 69:061509, 2004.

[99] N. W. Ashcroft and N. D. Mermin. Solid State Physics. Holt, Rinehart and Wiston, New York, 1976.

[100] P. Gillet, P. Richet, F. Guyot, and G. Fiquet. High-temperature thermodynamic properties of forsterite. J. Geophys. Res., 96:11805-11816, 1991.

[101] B. K. Sharma. Nonlinearity acoustical parameter and its relation with rao's acoustical parameter of liquid state. J. Acoust. Soc. Am., 73:106-109, 1983.

[102] B. Reynard, G. D. Price, and P. Gillet. Thermodynamic and anharmonic properties of forsterite, 
$\alpha-m g_{2} \operatorname{sio}_{4}$ : Computer modelling versus high-pressure and high-temperature measurements. $J$. Geophys. Res., 97:19791-19801, 1992.

[103] M. Ravi Kumar, R. R. Reddy, T. V. R. Rao, and B. K. Sharma. Thermoacoustical parameters of polymers at low temperatures. J. Appl. Polym. Sci., 51:1805-1815, 1994.

[104] R. W. Warfield. The gruneisen constant of polymers. Die Makromolekulare Chemie, 175:3285-3297, 1974.

[105] Y. Wada, A. Itani, T. Nishi, and S. Nagai. Gruneisen constant and thermal properties of crystalline and glassy polymers. J. Polym. Sci. Part A-2: Polym. Phys., 7:201-208, 1969.

[106] J. G. Kurro. Calculation of grüneisen parameters of polymers. J. Chem. Phys., 58:374-380, 1973.

[107] Yuli K. Godovsky. Thermophysical Properties of Polymers. Springer-Verlag, Berlin, 1992.

[108] J. K. Krüger, K.-P. Bohn, M. Pietralla, and J Schreiber. Anharmonic properties of fragile and strong liquids: temperature dependence of the mode grüneisen parameter. J. Phys.: Condens. Matter, 8:10863-10874, 1996.

[109] Edward M. Brody, Cathie J. Lubell, and Charles L. Beatty. Measurement of the grüneisen number in polystyrene by brillouin light scattering. J. Polym. Sci. : Polym. Phys., 13:295-301, 1975.

[110] E. Kato. Brillouin scattering in polymethyl methacrylate from 4 to $300 \mathrm{k}$ : Temperature dependence of the gruneisen constant and thermal properties. J. Chem. Phys., 73:1020-1025, 1980.

[111] Tian Lan, Xiaoli Tang, and Brent Fultz. Phonon anharmonicity of rutile tio2 studied by raman spectrometry and molecular dynamics simulations. Phys. Rev. B, 85:094305, 2012.

[112] L. D. Landau and E. M. Lifshitz. Course of Theoretical Physics, volume 1, Mechanics. ButterworthHeinemann, Oxford, iii edition, 1981.

[113] U. W. Gedde. Polymer Physics. Chapman and Hall, London, London, 1995.

[114] Koichi Fujisawa, Tomoo Shiomi, Fumiyuki Hamada, and Akio Nakajima. Van der waals-like equations of state. i. application to pure liquids. Polym. J., 13:993-1002, 1981.

[115] A. F. M. Barton. Internal pressure a fundamental liquid property. J. Chem. Educ., 48:156-162, 1971.

[116] Y. Marcus. Internal pressure of liquids and solutions. Chem. Rev., 113:6536-6551, 2013.

[117] K. Trachenko and V. V. Brazhkin. Heat capacity at the glass transition. Phys. Rev. B, 83:014201, 2011.

[118] Ivan Saika-Voivod, P. H. Poole, and F. Sciortino. Fragile-to-strong transition and polyamorphism in the energy landscape of liquid silica. Nature, 412:514-517, 2001.

[119] E. I. Andritsos, E. Zarkadoula, A. E. Phillips, M. T. Dove, C. J. Walker, V. V. Brazhkin, and K. Trachenko. The heat capacity of matter beyond the dulong-petit value. J. Phys.: Condens. Matter, 25:235401, 2013.

[120] D. H. Chung. Birch's law: Why is it so good? Science, 177:261-263, 1972.

[121] L. H. Sperling. Introduction to Physical Polymer Science. Wiley, Hoboken, 2006.

[122] John S. Harmon, Marios D. Demetriou, and William L. Johnson. Rheology and ultrasonic properties of pt57.5ni5.3cu14.7p22.5 liquid. Appl. Phys. Lett., 90:171923, 2007.

[123] J. Baschnagel and F. Varnik. Computer simulations of supercooled polymer melts in the bulk and in confined geometry. J. Phys.: Condens. Matter, 17:R851-R953, 2005.

[124] G. S. Grest and K. Kremer. Molecular dynamics simulation for polymers in the presence of a heat bath. Phys. Rev. A, 33(5):3628-3631, 1986.

[125] R. Mauri and D. Leporini. Violation of the fluctuation-dissipation theorem in confined driven colloids. Europhys. Lett., 76:1022-1028, 2006.

[126] S. Plimpton. Fast parallel algorithms for short-range molecular dynamics. J. Comput. Phys., 117:1-19, 1995.

[127] M. Doi and S. F. Edwards. The Theory of Polymer Dynamics. Clarendon Press, Oxford, 1988.

[128] R. Zwanzig and R. Mountain. High-frequency elastic moduli of simple fluids. J. Chem. Phys., 43:44644471, 1965.

[129] W. Götze. Complex Dynamics of Glass-Forming Liquids: A Mode-Coupling Theory. Oxford University Press, Oxford, 2008.

[130] A. E. Likhtman, S. K. Sukumaran, and J. Ramirez. Linear viscoelasticity from molecular dynamics simulation of entangled polymers. Macromolecules, 40:6748-6757, 2007. 
[131] M. P. Allen and D. J. Tildesley. Computer simulations of liquids. Oxford university press, Clarendon, 1987.

[132] Arno A. Veldhorst, Jeppe C. Dyre, and Thomas B. Schrøder. Scaling of the dynamics of flexible lennardjones chains. J. Chem. Phys., 141:054904, 2014.

[133] Joachim Buchholz, Wolfgang Paul, Fathollah Varnik, and Kurt Binder. Cooling rate dependence of the glass transition temperature of polymer melts: Molecular dynamics study. J. Chem.Phys., 117:73647372, 2002.

[134] R. Casalini and C. M. Roland. Density scaling of the structural and johari-goldstein secondary relaxations in poly(methyl methacrylate). Macromolecules, 46(15):6364-6368, 2013.

[135] A. Monaco. Study of the vibrational dynamics of glasses. PhD thesis, Universitè Joseph-Fourier, Grenoble (France), 2006.

[136] A. Patkowski, W. Steffen, H. Nilgens, E. W. Fischer, and R. Pecora. Depolarized dynamic light scattering from three low molecular weight glass forming liquids: A test of the scattering mechanism. J. Chem. Phys., 106:8401-8408, 1997.

[137] R. E. Gibson and O. H. Loeffler. Pressure-volume-temperature relations in solutions. ii. the energyvolume coefficients of aniline, nitrobenzene, bromobenzene and chlorobenzene. J. Amer. Chem. Soc., 61:2515-2522, 1939.

[138] C. M. Roland and R. Casalini. Effect of chemical structure on the isobaric and isochoric fragility in polychlorinated biphenyls. J. Chem. Phys., 122(13):134505, 2005.

[139] M. Paluch, R. Casalini, and C. M. Roland. Relative contributions of thermal energy and free volume to the temperature dependence of structural relaxation in fragile glass-forming liquids. Phys. Rev. B, 66:092202, 2002.

[140] S. Hensel-Bielowka, J. Ziolo, M. Paluch, and C. M. Roland. The effect of pressure on the structural and secondary relaxations in 1,1â-bis (p-methoxyphenyl) cyclohexane. J. Chem. Phys., 117(5):2317-2323, 2002.

[141] K. Kaminski, S. Maslanka, J. Ziolo, M. Paluch, K. J. McGrath, and C. M. Roland. Dielectric relaxation of $\alpha$-tocopherol acetate (vitamin e). Phys. Rev. E, 75:011903, 2007.

[142] R. Casalini and C. M. Roland. Scaling of the supercooled dynamics and its relation to the pressure dependences of the dynamic crossover and the fragility of glass formers. Phys. Rev. B, 71:014210, 2005.

[143] R. Casalini, M. Paluch, , and C. M. Roland. Dynamics of salol at elevated pressure. J. Phys. Chem. A, 107(13):2369-2373, 2003.

[144] C. M. Roland and R. Casalini. Dynamics of poly(cyclohexyl methacrylate): neat and in blends with poly(-methylstyrene). Macromolecules, 40:3631-3639, 2007.

[145] R. Casalini, C. M. Roland, and S. Capaccioli. Effect of chain length on fragility and thermodynamic scaling of the local segmental dynamics in poly(methylmethacrylate). J. Chem. Phys., 126:184903, 2007.

[146] Marian Paluch, Riccardo Casalini, Andreas Best, and Adam Patkowski. Volume effects on the molecular mobility close to glass transition in supercooled phenylphthalein-dimethylether. ii. J. Chem. Phys., 117(16):7624-7630, 2002.

[147] D. Gundermann, U. R. Pedersen, T. Hecksher, N. P. Bailey, B. Jakobsen, T. Christensen, N. B. Olsen, T. B. Schroder, D. Fragiadakis, R. Casalini, C. M. Roland, J. C. Dyre, and K. Niss. Predicting the density-scaling exponent of a glass-forming liquid from prigogine-defay ratio measurements. Nat Phys, 7:816-821, 2011.

[148] R. Casalini and C. M. Roland. Temperature and density effects on the local segmental and global chain dynamics of poly(oxybutylene). Macromolecules, 38(5):1779-1788, 2005.

[149] G. Floudas and T. Reisinger. Pressure dependence of the local and global dynamics of polyisoprene. $J$. Chem. Phys., 111(11):5201-5204, 1999.

[150] C. M. Roland, M. Paluch, and R. Casalini. Effects of the volume and temperature on the global and segmental dynamics in poly(propylene glycol) and 1,4-polyisoprene. Journal of Polymer Science Part B: Polymer Physics, 42(23):4313-4319, 2004. 
[151] C. M. Roland and R. Casalini. Temperature and volume effects on local segmental relaxation in poly(vinyl acetate). Macromolecules, 36:1361-1367, 2003.

[152] M. Paluch, C. M. Roland, and S. Pawlus. Temperature and pressure dependence of the $\alpha$-relaxation in polymethylphenylsiloxane. J. Chem. Phys., 116:10932, 2002.

[153] Motosuke. Naoki, Hideki. Endou, and Katsuhiro. Matsumoto. Pressure effects on dielectric relaxation of supercooled ortho-terphenyl. J. Phys. Chem., 91(15):4169-4174, 1987.

[154] Motosuke Naoki and Susumu Koeda. Pressure-volume-temperature relations of liquid, crystal, and glass of o-terphenyl: excess amorphous entropies, and factors determining molecular mobility. J. Phys. Chem., 93(2):948-955, 1989.

[155] C. Hansen, F. Stickel, T. Berger, R. Richert, and E. W. Fischer. Dynamics of glass-forming liquids. iii. comparing the dielectric $\hat{\imath} \pm$ - and $\hat{1}$-relaxation of 1-propanol and o-terphenyl. J. Chem. Phys., 107(4):1086-1093, 1997.

[156] Simone Capaccioli, Giancarlo Ruocco, and Francesco Zamponi. Dynamically correlated regions and configurational entropy in supercooled liquids. J. Phys. Chem. B, 112(34):10652-10658, 2008.

[157] A. Tölle. Neutron scattering studies of the model glass former ortho -terphenyl. Rep. Prog. Phys., 64:1473, 2001.

[158] A. Tölle, H. Schober, J. Wuttke, O. G. Randl, and F. Fujara. Fast relaxation in a fragile liquid under pressure. Phys. Rev. Lett., 80:2374-2377, 1998.

[159] Günter Dlubek, Muhammad Q. Shaikh, Klaus Rätzke, Jürgen Pionteck, Marian Paluch, and Franz Faupel. Subnanometre size free volumes in amorphous verapamil hydrochloride: A positron lifetime and \{PVT\} study through tg in comparison with dielectric relaxation spectroscopy. European Journal of Pharmaceutical Sciences, 41(2):388 - 398, 2010.

[160] Z. Wojnarowska, K. Grzybowska, A. Grzybowski, M. Paluch, K. Kaminski, P. Wlodarczyk, K. Adrjanowicz, and J. Pionteck. Study of molecular dynamics of the pharmaceutically important protic ionic liquid verapamil hydrochloride. ii. test of entropic models. J. Chem. Phys., 132(9):094506, 2010.

[161] M. Paluch, C. M. Roland, J. Gapinski, and A. Patkowski. Pressure and temperature dependence of structural relaxation in diglycidylether of bisphenol a. The Journal of Chemical Physics, 118(7):31773186, 2003.

[162] Simone Capaccioli, Daniele Prevosto, Mauro Lucchesi, Masoud Amirkhani, and Pierangelo Rolla. Relation between configurational entropy and relaxation dynamics of glass-forming systems under volume and temperature reduction. J. Non-Cryst. Solids, 355(10-12):753 - 758, 2009.

[163] M. Paluch, S. Pawlus, and C. M. Roland. Pressure and temperature dependence of the $\alpha$-relaxation in poly(methyltolylsiloxane). Macromolecules, 35:7338-7342, 2002.

[164] C. M. Roland and R. Casalini. Comment on: "disentangling density and temperature effects in the viscous slowing down of glass forming liquids" [j. chem. phys. [bold 120], 6135 (2004)]. J. Chem. Phys., 121:11503-11504, 2004.

[165] R. Casalini, S. Capaccioli, M. Lucchesi, P. A. Rolla, M. Paluch, S. Corezzi, and D. Fioretto. Effect of pressure on the dynamics of glass formers. Phys. Rev. E, 64:041504, 2001.

[166] S. Corezzi, M. Beiner, H. Huth, K. SchrÃter, S. Capaccioli, R. Casalini, D. Fioretto, and E. Donth. Two crossover regions in the dynamics of glass forming epoxy resins. J. Chem. Phys., 117(5):2435-2448, 2002.

[167] S. Pawlus, R. Casalini, C. M. Roland, M. Paluch, S. J. Rzoska, and J. Ziolo. Temperature and volume effects on the change of dynamics in propylene carbonate. Phys. Rev. E, 70:061501, 2004.

[168] R. Casalini and C. M. Roland. Dynamic properties of polyvinylmethylether near the glass transition. $J$. Chem. Phys., 119:4052-4059, 2003.

[169] K. L. Ngai, J. Habasaki, D. Prevosto, S. Capaccioli, and Marian Paluch. Thermodynamic scaling of relaxation time and viscosity stems from the johari-goldstein -relaxation or the primitive relaxation of the coupling model. J. Chem. Phys., 137(3):034511, 2012.

[170] C. M. Roland, T. Psurek, S. Pawlus, and M. Paluch. Segmental- and normal-mode dielectric relaxation of poly(propylene glycol) under pressure. J. Polym. Sci., Part B: Polym. Phys., 41:3047-3052, 2003. 
[171] Jiaxi Guo and Sindee L. Simon. Thermodynamic scaling of polymer dynamics versus $\mathrm{t}-\mathrm{tg}$ scaling. $J$. Chem. Phys., 135(7):074901, 2011.

[172] S. Hensel-Bielowka and M. Paluch. Origin of the high-frequency contributions to the dielectric loss in supercooled liquids. Phys. Rev. Lett., 89:025704, 2002.

[173] R. Casalini, R. F. Gamache, and C. M. Roland. Density-scaling and the prigogine-defay ratio in liquids. J. Chem. Phys., 135(22):224501, 2011.

[174] C.A. Angell. Relaxation in liquids, polymers and plastic crystals - strong/fragile patterns and problems. J.Non-Crystalline Sol., 131-133:13-31, 1991. 